Notre Dame Journal of Formal Logic

Volume 48, Number 1, 2007

\title{
Bounds on Weak Scattering
}

\author{
Gerald E. Sacks
}

In Memory of Jon Barwise

\begin{abstract}
The notion of a weakly scattered theory $T$ is defined. $T$ need not be scattered. For each $\mathcal{A}$ a model of $T$, let $\operatorname{sr}(\mathcal{A})$ be the Scott rank of $\mathcal{A}$. Assume $\operatorname{sr}(\mathcal{A}) \leq \omega_{1}^{\mathcal{A}}$ for all $\mathcal{A}$ a model of $T$. Let $\sigma_{2}^{T}$ be the least $\Sigma_{2}$ admissible ordinal relative to $T$. If $T$ admits effective $\mathrm{k}$-splitting as defined in this paper, then $\exists \theta<\sigma_{2}^{T}$ such that $\operatorname{sr}(\mathcal{A})<\theta$ for all $\mathscr{A}$ a model of $T$.
\end{abstract}

\section{Introduction}

This paper has two themes less disparate than they seem at first reading:

1. extending classical descriptive set theoretic results that impose bounds on suitably defined functions from $\omega^{\omega}$ into $\omega_{1}$;

2. extending and clarifying some early results on Scott ranks of countable structures sketched in [15]. ${ }^{1}$

Let $F$ be a function, possibly partial, from $\omega^{\omega}$ into $\omega_{1}$. A typical classical bounding theorem says the range of $F$ is bounded by a countable ordinal if the graph of $F$ has a suitable definition. For example, the graph of $F$ is $\Sigma_{1}^{1}$ with real parameter $p$; in this formulation the graph of $F$ is viewed as a subset of $\omega^{\omega} \times \omega_{1}$ by requiring each value of $F$ to be a well ordering of $\omega$. Let $F(X)$ ambiguously denote the well ordering and also the ordinal represented by the well ordering. For each $X, F(X)$ is the unique solution of a $\Sigma_{1}^{1}$ formula with parameters $p, X$. Consequently $F(X)$ (the well ordering) is hyperarithmetic in $p, X$, and so

$$
F(X)<\omega_{1}^{p, X},
$$

Received August 8, 2005; accepted November 14, 2005; printed February 26, 2007

2000 Mathematics Subject Classification: Primary, 03C70, 03D60

Keywords: weakly scattered theories, bounds on Scott rank

(C) 2007 University of Notre Dame 
the least ordinal not recursive in $p, X$. The effective version of the theorem says that the bound on the range of $F$ is an ordinal less than $\omega_{1}^{p}$.

A recursion-theoretic approach to the effective bound originated by Kleene is as follows. (See Sacks [16] for details.) Suppose

$$
\left(\forall \gamma<\omega_{1}^{p}\right)(\exists X)[F(X)>\gamma] .
$$

Let $R_{e}^{p}$ be the linear ordering of $\omega$ recursive in $p$ via index $e$. Define $W^{p}$ to be the set of all $e$ such that $R_{e}^{p}$ is a well ordering. Then

$e \in W^{p} \longleftrightarrow \exists X \exists g\left[g\right.$ is a 1-1 order-preserving map of $R_{e}^{p}$ into $\left.F(X)\right]$.

But then $W^{p}$ is $\Sigma_{1}^{1}$ with parameter $p$. This last is false according to a Kleene hierarchy result that says $W^{p}$ is universal $\Pi_{1}^{1}$ with parameter $p$, hence not $\Sigma_{1}^{1}$ with parameter $p$.

A model theoretic approach to effective bounds is the path taken in this paper. A sketch may help to clarify later sections. Let $A(p)$ be the least $\Sigma_{1}$ admissible set with $p$ as a member. Let $Z$ be a $\Sigma_{1}^{A(p)}$ definable set of sentences of $\mathcal{L}_{\omega_{1}, \omega}$ coded by elements of $A(p)$ such that every model $M$ of $Z$ has the following properties:

1. The ordinals recursive in $p$ form a proper initial segment of the ordinals in the sense of $M$.

2. There is an $X_{0} \in M$ such that for all $\gamma<\omega_{1}^{p}, F\left(X_{0}\right)>\gamma$.

3. $p \in M$ and $M$ is a $\Sigma_{1}$ admissible structure.

Assume the range of $F$ is not bounded by an ordinal below $\omega_{1}^{p}$. Then each $A(p)$ finite subset of $Z$ (i.e., each subset of $Z$ that is a member of $A(p)$ ) is consistent, and so $Z$ has a model by Barwise Compactness. With the addition of "effective" type omitting, as in Grilliot [5] or Keisler [7], $Z$ has a model $M$ that omits $\omega_{1}^{p}$ but has nonstandard ordinals greater than all standard ordinals less than $\omega_{1}^{p}$. Then

$$
\omega_{1}^{p, X_{0}} \leq \omega_{1}^{p}
$$

otherwise, $\omega_{1}^{p}$ is recursive in $\left\langle p, X_{0}\right\rangle$ and so $\omega_{1}^{p} \in M$. But then $\omega_{1}^{p, X_{0}}=\omega_{1}^{p}$ and $F\left(X_{0}\right) \geq \omega_{1}^{p, X_{0}}$ by property (2) of $Z$, which contradicts (1.1).

The search for a bounding theorem that extends the classical result seems hopeless at first. An extension has to talk about an $F$ that allows $F(X) \geq \omega_{1}^{X, p}$, but $\omega_{1}^{X, p}$, as a function of $X$, is unbounded. Model theory comes to the rescue. Every countable structure $\mathcal{A}$ has a Scott rank [17], $\operatorname{sr}(\mathcal{A})$, an ordinal that can be as high as $\omega_{1}^{\mathscr{A}}+1$ (see Section 2 for elaboration).

Let $T$ be a countable theory. A reasonable starting assumption on $T$ is

$$
\forall \mathcal{A}\left[\mathcal{A} \models T \longrightarrow \operatorname{sr}(\mathcal{A}) \leq \omega_{1}^{\mathcal{A}}\right] .
$$

An ingenious example (MA) devised by Makkai [11] shows that (1.5) is not enough. Examination of (MA) and its illuminative extensions in Knight and Young [8] leads to two further assumptions on $T$. The first, effective $k$-splitting, is technical and perhaps peripheral and is discussed further in Sections 9 and 10. The second, weakly scattered, is central. The theory $T_{M}$ associated with (MA) satisfies (1.5) and has properties similar to effective k-splitting. In addition for every $\Sigma_{1}$ admissible countable $\alpha, T_{M}$ has a model $\mathcal{A}$ such that

$$
\omega_{1}^{\mathcal{A}}=\alpha=\operatorname{sr}(\mathcal{A}) .
$$


Corollary 9.5 says if $T$ is weakly scattered, satisfies (1.5), and has effective ksplitting, then there is a countable bound on the Scott ranks of the countable models of $T$; the effective version provides a bound less than the first $\Sigma_{2}$ admissible ordinal relative to $T$ in contrast to the classical case above, where the effective bound on the range of $F$ is less than $\omega_{1}^{p}$, the first $\Sigma_{1}$ admissible ordinal relative to $p$.

The notion of weakly scattered is inspired by Morley's concept of scattered. Let $\mathscr{L}$ be a countable first-order language, $\mathscr{L}_{0}$ a countable fragment of $\mathscr{L}_{\omega_{1}, \omega}$, and $T \subseteq \mathscr{L}_{0}$ a theory (i.e., a set of sentences) with a model. For (a) and (b) below, let $\mathcal{L}^{\prime}$ be any countable fragment of $\mathcal{L}_{\omega_{1}, \omega}$ extending $\mathscr{L}_{0}$, and $T^{\prime}$ any finitarily consistent, $\omega$-complete theory contained in $\mathcal{L}^{\prime}$ and extending $T$. (The notions of finitary consistency and $\omega$-completeness for fragments are reviewed at the beginning of Section 4.) $T$ is said to be scattered if and only if (a) and (b) hold.

(a) For all $n>0$ and all $T^{\prime}, S_{n} T^{\prime}$, the set of all $n$-types over $T^{\prime}$, is countable.

(b) For all $\mathscr{L}^{\prime}$, the set $\left\{T^{\prime} \mid T^{\prime} \subseteq \mathcal{L}^{\prime}\right\}$ is countable.

The above definition of scattered is equivalent to the one in Morley's groundbreaking [13]. $T$ is said to be weakly scattered if and only if (a) holds. By [13], a scattered theory can have at most $\omega_{1}$ many countable models. In contrast, a weakly scattered theory can have $2^{\omega}$ many countable models.

Knight [9] has announced a counterexample to Vaught's Conjecture (VC), a scattered first-order theory with $\omega_{1}$ many countable models. VC has a precise formulation in Section 5.

In [15] the following bounding result was established: if $T$ is scattered and satisfies (1.5), then $T$ has only countably many countable models; furthermore, every countable model of $T$ has a countable copy in $L(\beta, T)$ for some $\beta<\sigma_{2}^{T}$, the least $\alpha$ such that $L(\alpha, T)$ is $\Sigma_{2}$ admissible. Hence Vaught's Conjecture holds for $T$ if $T$ satisfies (1.5). The proofs given in [15] were somewhat sketchy, so missing details needed in later sections of this paper are given in Sections 3 through 5. If Vaught's Conjecture is false, then results for scattered theories yield information about models of counterexamples to VC. Theorem 4.9(vii) says if VC fails for $T$, then $T$ has a model of cardinality $\omega_{1}$ not elementarily equivalent in the sense of $\mathcal{L}_{\omega_{1}, \omega}$ to any countable model (Harnik and Makkai [6]). Theorem 5.3 describes an $\omega_{1}$-sequence of atomic and saturated models that every counterexample must possess. Section 5 includes a related absoluteness result implicit in Morley [13]: $\mathrm{VC}(T)$, Vaught's Conjecture for $T$, is a $\Sigma_{1}^{L\left(\omega_{1}^{L(T)}, T\right)}$ predicate of $T$, hence, $\Sigma_{2}^{1}$.

Steel [18], as reported in [11], used an assumption stronger than (1.5) to prove $\operatorname{VC}(T)$. In Section 2 an arbitrary countable structure $\mathcal{A}$ is associated with a theory $T_{\omega_{1}^{\mathcal{A}}}^{\mathcal{A}}$ contained in a countable fragment of $\mathcal{L}_{\omega_{1}, \omega}$ canonically generated from $\mathcal{A}$. By an argument of Nadel [14], $\mathcal{A}$ is a homogeneous model of $T_{\omega_{1}^{\mathcal{A}}}^{\mathcal{A}}$. Steel's assumption is equivalent to 'for every $\mathcal{A}$ a model of $T, T_{\omega_{1}^{\mathcal{A}}}^{\mathcal{A}}$ is $\omega$-categorical'. Assumption (1.5) is equivalent to 'for every $\mathcal{A}$ a model of $T, \mathcal{A}$ is an atomic model of $T_{\omega_{1}^{A}}^{\mathcal{A}}$ '. Sacks and Young [12] produced a structure $\mathcal{A}$ such that $\mathcal{A}$ is an atomic model of $T_{\omega_{1}^{\mathcal{A}}}^{\mathcal{A}}$, but $T_{\omega_{1}^{\mathcal{A}}}^{\mathcal{A}}$ is not $\omega$-categorical. (In addition, $\omega_{1}^{\mathcal{A}}=\omega_{1}^{C K}$ and $T_{\omega_{1}^{\mathcal{A}}}^{\mathcal{A}}$ is a $\Delta_{1}$ subset of $L\left(\omega_{1}^{C K}\right)$.)

Sections 7 through 9 are devoted to bounding for weakly scattered theories. 


\section{Scott Analysis and Rank}

This section revisits [15] as promised in Section 1. Scott [17] showed that an arbitrary countable structure $\mathcal{A}$ with underlying first-order language $\mathcal{L}$ can be characterized up to isomorphism by a single sentence of $\mathcal{L}_{\omega_{1}, \omega}$. In essence there is a countable fragment $\mathcal{L}^{\mathcal{A}}$ of $\mathcal{L}_{\omega_{1}, \omega}$ such that $\mathcal{A}$ is the atomic model of $T^{\mathcal{A}}$, the complete theory of $\mathcal{A}$ in $\mathcal{L}^{\mathcal{A}}$. Nadel [14] pointed the way to a canonical choice for $\mathcal{L}^{\mathcal{A}}$.

The admissible set $L\left(\omega_{1}^{\mathcal{A}}, \mathcal{A}\right)$ is Gödel's $L$ relativized to $\mathcal{A}$ as an element ${ }^{2}$ and chopped off at $\omega_{1}^{\mathcal{A}}$, the least $\gamma$ such that $L(\gamma, \mathcal{A})$ is $\Sigma_{1}$ admissible. Let

$$
\mathscr{L}_{\omega_{1}^{\mathcal{A}}, \omega}^{\mathcal{A}}=\mathcal{L}_{\omega_{1}, \omega} \cap L\left(\omega_{1}^{\mathcal{A}}, \mathcal{A}\right) \text {. }
$$

Nadel [14] showed that

$$
\mathcal{A} \text { is a homogeneous model of its complete theory } T_{\omega_{1}^{\mathcal{A}}, \omega}^{\mathcal{A}} \text { in } \mathcal{L}_{\omega_{1}^{\mathcal{A}}, \omega}^{\mathcal{A}} \text {. }
$$

It follows that $\mathcal{A}$ is the atomic model of its complete theory in

$$
\mathcal{L}_{\omega_{1}, \omega} \cap L\left(\omega_{1}^{\mathcal{A}}+1, \mathcal{A}\right),
$$

since the types over $T_{\omega_{1}^{\mathcal{A}}, \omega}^{\mathcal{A}}$ realized in $\mathcal{A}$ are first-order definable over $L\left(\omega_{1}^{\mathcal{A}}, \mathcal{A}\right)$ and so become atoms of the complete theory of $\mathcal{A}$ contained in (2.3).

A $\Sigma_{1}$ recursion defines a canonical choice for $\mathcal{L}^{\mathcal{A}}$ and yields the definition of Scott rank for $\mathcal{A}$.

1. $\mathcal{L}_{0}^{\mathcal{A}}=\mathcal{L}$.

2. $\mathcal{L}_{\lambda}^{\mathcal{A}}=\cup\left\{\mathcal{L}_{\delta}^{\mathcal{A}} \mid \delta<\lambda\right\}$ for limit $\lambda$.

3. $T_{\delta}^{\mathcal{A}}=$ complete theory of $\mathcal{A}$ in $\mathcal{L}_{\delta}^{\mathcal{A}}$.

4. $\mathcal{L}_{\delta+1}^{\mathcal{A}}=$ least fragment $\mathcal{L}^{+}$of $\mathcal{L}_{\omega_{1}, \omega}$ such that $\mathcal{L}^{+} \supseteq \mathcal{L}_{\delta}^{\mathcal{A}}$, and for each $n>0$, if $p(\vec{x})$ is a nonprincipal $n$-type of $T_{\delta}^{\mathcal{A}}$ realized in $\mathcal{A}$, then the conjunction

$$
\wedge\{\mathcal{F}(\vec{x}) \mid \mathcal{F}(\vec{x}) \in p(\vec{x})\}
$$

is a member of $\mathcal{L}^{+}$.

Note that if $\mathcal{A}$ is isomorphic to $\mathcal{B}$, then $\mathcal{L}_{\delta}^{\mathcal{A}}=\mathcal{L}_{\delta}^{\mathscr{B}}$ and $T_{\delta}^{\mathcal{A}}=T_{\delta}^{\mathcal{B}}$ for all $\delta$. For some $\delta<\omega_{1}$, all the $n$-types of $T_{\delta}^{\mathcal{A}}$ realized in $\mathcal{A}$ are principal. To see this, fix $\gamma$ and suppose some nonprincipal type $p_{\gamma+1}$ of $T_{\gamma+1}^{\mathcal{A}}$ is realized in $\mathcal{A}$. Let $p_{\gamma}$ be the restriction of $p_{\gamma+1}$ to $T_{\gamma}^{A}$. Since $p_{\gamma+1}$ is nonprincipal, there is a formula $g(\vec{x})$ of $\mathcal{L}_{\gamma+1}^{\mathcal{A}}$ such that both

$$
\exists \vec{x}\left[p_{\gamma}(\vec{x}) \wedge g(\vec{x})\right] \text { and } \exists \vec{x}\left[p_{\gamma}(\vec{x}) \wedge \neg g(\vec{x})\right]
$$

belong to $T_{\gamma+1}^{\mathcal{A}}$. Then there are $n$-tuples $\vec{b}$ and $\vec{c}$ of $\mathcal{A}$ such that

$$
\mathcal{A} \models\left[p_{\gamma}(\vec{b}) \wedge \mathcal{g}(\vec{b})\right] \text {, and } \mathcal{A} \models\left[p_{\gamma}(\vec{c}) \wedge \neg \mathcal{G}(\vec{c})\right] \text {. }
$$

Thus a distinction between $\vec{b}$ and $\vec{c}$ is made by a formula of $\mathcal{L}_{\gamma+1}^{\mathcal{A}}$ but not by any formula of $\mathcal{L}_{\gamma}^{\mathcal{A}}$. Since $\mathcal{A}$ is countable, only countably many distinctions can be made.

Let $d_{\mathcal{A}}$ be the the least $\delta<\omega_{1}$ such that every distinction ever made is made by a formula of $\mathcal{L}_{\delta}^{\mathcal{A}}$. Then

$$
\mathcal{A} \text { is the atomic model of } T_{d_{\mathcal{A}}+1}^{\mathcal{A}} \text {. }
$$


The Scott Rank of $\mathcal{A}$ is defined by

$$
\operatorname{sr}(\mathcal{A})=\text { least } \alpha\left[\mathcal{A} \text { is the atomic model of } T_{\delta}^{A}\right] .
$$

If $\mathcal{A}$ is isomorphic to $\mathscr{B}$, then $\operatorname{sr}(\mathcal{A})=\operatorname{sr}(\mathscr{B})$. Nadel's proof of (2.2), p. 273 of [14], sketched below, also shows

$$
\mathcal{A} \text { is a homogeneous model of } T_{\omega_{1}^{\mathcal{A}}}^{\mathcal{A}} \text {. }
$$

Hence $d_{\mathcal{A}} \leq \omega_{1}^{\mathcal{A}}$, and so

$$
\operatorname{sr}(\mathcal{A}) \leq \omega_{1}^{\mathcal{A}}+1
$$

Note that $\mathcal{L}_{\delta}^{\mathcal{A}}$ and $T_{\delta}^{\mathcal{A}}$, as functions of $\delta<\omega_{1}^{\mathcal{A}}$, are $\Sigma_{1}^{L\left(\omega_{1}^{\mathcal{A}}, \mathcal{A}\right)}$; that is, their graphs are $\Sigma_{1}$ definable subsets of $L\left(\omega_{1}^{\mathcal{A}}, \mathcal{A}\right)$. Since the formulas of $\mathcal{L}_{\omega_{1}^{\mathcal{A}}}^{\mathcal{A}}$ and $T_{\omega_{1}^{\mathcal{A}}}^{\mathcal{A}}$ are "enumerated" in increasing order of complexity,

$$
\mathcal{L}_{\omega_{1}^{\mathcal{A}}}^{\mathcal{A}} \text { and } T_{\omega_{1}^{\mathcal{A}}}^{\mathcal{A}} \text { are } \Delta_{1}^{L\left(\omega_{1}^{\mathcal{A}}, \mathcal{A}\right)} \text {. }
$$

To prove (2.6), let $p(\vec{x})$ be an $n$-type, and $q(\vec{x}, y)$ an $(n+1)$-type, of $T_{\omega_{1}^{\mathcal{A}}}^{\mathcal{A}}$, and $\vec{a}, \vec{b} n$-tuples of $\mathcal{A}$. Suppose $p(\vec{x}) \subseteq q(\vec{x}, y)$ and

$$
\mathcal{A} \models[p(\vec{a}) \wedge p(\vec{b}) \wedge \exists y q(\vec{a}, y)] .
$$

For homogeneity, a $d \in \mathcal{A}$ is required so that $\mathcal{A} \models q(\vec{b}, d)$. Suppose no such $d$ exists. Let $q_{\delta}(x, y)$ be the restriction of $q(x, y)$ to $\mathcal{L}_{\delta}^{\mathcal{A}}$. Then

$$
\left\{q_{\delta}(x, y) \mid \delta<\omega_{1}^{\mathcal{A}}\right\} \text { is } \Sigma_{1}^{L\left(\omega_{1}^{\mathcal{A}}, \mathcal{A}\right)} .
$$

For each $d \in \mathcal{A}$, there is a $\delta<\omega_{1}^{\mathcal{A}}$ such that $\mathcal{A} \models \neg q_{\delta}(\vec{b}, d)$. Since $\delta$ can be defined as a $\Sigma_{1}^{L\left(\omega_{1}^{\mathcal{A}}, \mathcal{A}\right)}$ function of $d$, the $\Sigma_{1}$ admissibility of $L\left(\omega_{1}^{\mathcal{A}}, \mathcal{A}\right)$ implies there is a $\delta_{\infty}<\omega_{1}^{\mathcal{A}}$ such that $\mathcal{A}=\forall y \neg q_{\delta_{\infty}}(\vec{b}, y)$. But then

$$
\mathcal{A} \models \forall y \neg q(\vec{a}, y) .
$$

A typical use of Scott rank in conjunction with Barwise Compactness and Grilliot type omitting is as follows.

Proposition 2.1 Suppose $L(\alpha, T)$ is countable and $\Sigma_{1}$ admissible. If for each $\beta<\alpha, T$ has a model of Scott rank $\geq \beta$, then $T$ has a countable model such that

$$
\operatorname{sr}(\mathcal{A}) \geq \omega_{1}^{T, \mathcal{A}}=\alpha .
$$

Note that the $\mathcal{A}$ of (2.12) must have Scott rank either $\alpha$ or $\alpha+1$ by (2.7). Forcing the outcome to be $\alpha+1$ is a problem addressed in this paper but far from resolved.

\section{Small $\Delta_{0}^{Z F}$ Sets}

The following is one of many variations (e.g., Makkai [10]) on a theme initiated by Barwise [2], an extension of a recursion theoretic fact needed for the enumeration of models of both scattered and weakly scattered theories. The variation below was mentioned and used in [15]. The recursion theoretic fact is as follows: if a set $S$ of reals is $\Sigma_{1}^{1}$ and has cardinality less that $2^{\omega}$, then there exists a hyperarithmetic real $H$ such that every member of $S$ is Turing reducible to $H$; in addition, an index for $H$ can be computed uniformly from an index for $S$. The latter uniformity is 
key to establishing the $\Sigma_{1}$ character of the enumeration of models in Sections 4 and 8. Recall that a $\Delta_{0}^{Z F}$ formula is a formula in the language of set theory with only bounded quantifiers ' $(\forall x \epsilon y)$ ' and ' $(\exists u \epsilon v)$ '. Let $D(x, y)$ be a $\Delta_{0}^{Z F}$ lightface formula and $A$ a countable $\Sigma_{1}$ admissible set. Suppose $p, b \in A$. Define

$$
S_{p, b}=\{x \mid x \in V \wedge x \subseteq b \wedge D(x, p)\} .
$$

Theorem 3.1 If $S_{p, b} \notin A$, then the cardinality of $S_{p, b}$ is $2^{\omega}$.

Proof Let the language $\mathscr{L}$ consist of $\in$, bounded quantifiers $\forall x \in y$ and $\exists x \in y$, an individual constant $\underline{e}$ for each $e \in A$, and a special individual constant $\underline{c}$ different from all the $\underline{e}$ s. Let $Z$ be the following $\Delta_{1}^{A}$ set of sentences of $\mathcal{L}$.

1. The atomic diagram of $A: \underline{d} \in \underline{e} \leftrightarrow d \in e ; \underline{d} \notin \underline{e} \leftrightarrow d \notin e$ for $d, e \in A$.

2. $\underline{c} \subseteq \underline{b}, D(\underline{c}, \underline{p})$, and $\underline{c} \neq \underline{e}$ for all $e \in A$.

Suppose $Z$ is not consistent in the sense of $\mathscr{L}_{\omega_{1}, \omega}$. Then there is a $z_{0} \in A$ such that $z_{0} \subseteq Z$ and $z_{0}$ is not consistent. And $z_{0}$ consists of some $A_{0} \in A$ such that $A_{0}$ is a subset of the atomic diagram of $A$, and

$$
\underline{c} \subseteq \underline{b}, D(\underline{c}, \underline{p}) \text {, and }\{\underline{c} \neq \underline{e} \mid e \in f\}
$$

for some $f \in A$. Since $z_{0}$ is inconsistent, there is a deduction $E \in A$ of

$$
[\underline{c} \subseteq \underline{b} \wedge D(\underline{c}, \underline{p})] \longrightarrow \underline{c} \in f
$$

from $A_{0}$. But then $S_{p, b} \subseteq f$ and so $S_{p, b} \in A$.

Suppose $Z$ is consistent. Then a Henkin-style construction in $\omega$ many stages yields a model of $Z$, hence, an actual $c \in\left(S_{p, b}-A\right)$. At stage $j$, a sentence $\sigma$ of $\mathscr{L}$ is considered, and $\sigma_{j}$ is either $\sigma$ or $\neg \sigma$ so long as $Z \cup\left\{\sigma_{i} \mid i \leq j\right\}$ is consistent. If $\sigma_{j}$ is an infinite disjunction (e.g., $\sigma_{j}$ begins with ' $\exists x \in \underline{e}$ '), then some component of $\sigma_{j}$ is added immediately.

The construction can be varied so $2^{\omega}$ many $c \mathrm{~s}$ are produced. Let $t$ be a one-one map of $\omega$ onto $\{\underline{g} \mid g \in b\}$. After $\sigma_{j}$ is chosen, and before $\sigma_{j+1}$ is chosen, create a split as follows. Choose an $n$ so that $(t(n) \in \underline{c})$ and $(t(n) \notin \underline{c})$ are each consistent with $Z \cup\left\{\sigma_{i} \mid i \leq j\right\}$. Then the construction takes $2^{\omega}$ different paths, and different paths produce different $c$ s. Such splits always exist. Otherwise there is a $j$ such that $Z \cup\left\{\sigma_{i} \mid i \leq j\right\}$ is consistent and for each $n$ there is a deduction $D_{n} \in \mathcal{A}$ from $Z \cup\left\{\sigma_{i} \mid i \leq j\right\}$ of either $(t(n) \in \underline{c})$ or $(t(n) \notin c)$. The $\Sigma_{1}$ admissibility of $A$ puts all the $D_{n}$ s in some $D \in A$. $D$ decides which elements of $\underline{b}$ belong to $\underline{c}$. Hence there is an $e \in A$ such that $(\underline{c}=\underline{e})$ is deducible from $Z \cup\left\{\sigma_{i} \mid i \leq j\right\}$, a contradiction.

Corollary 3.2 $S_{p, b}$ is countable $\longleftrightarrow S_{p, b} \in A$.

Theorem 3.3 There exists a lightface $\Sigma_{1}^{Z F}$ formula $\mathcal{F}(u, v, w)$ such that for any countable $\Sigma_{1}$ admissible set $A$ and any $p, b, s \in A$,

$$
\begin{gathered}
S_{p, b} \text { is countable } \longrightarrow A \models \exists w \mathcal{F}(\underline{p}, \underline{b}, w) ; \\
(\forall s \in A)\left\{[A \models \mathcal{F}(\underline{p}, \underline{b}, \underline{s})] \longrightarrow s=S_{p, b}\right\} .
\end{gathered}
$$


Proof The existence of $\mathcal{F}$ is implicit in the proof of Theorem 3.1. Thus $Z$ is inconsistent if and only if $S_{p, b}$ is countable if and only if $S_{p, b} \in \mathcal{A}$. The statement

$$
A \models \mathcal{F}(\underline{p}, \underline{b}, \underline{s})
$$

says

(i) there exist $A_{0} \in A$ and $E$ such that $A_{0} \subseteq$ atomic diagram of $A$, and $E$ is a deduction of (3.3) from $A_{0}$; and

(ii)

$$
s=\{x \mid x \in f \wedge x \subseteq b \wedge D(x, p)\}
$$

\section{Enumeration of Models for Scattered Theories}

Let $\mathcal{L}_{0}$ be a countable fragment of $\mathcal{L}_{\omega_{1}, \omega}$ for some countable first-order language $\mathcal{L}$ and $T \subseteq \mathscr{L}_{0}$ a theory with a model. Throughout this section $T$ is scattered as defined in Section 1. For convenience assume $T$ mentions all formulas of $\mathscr{L}_{0}$; thus $\mathscr{L}_{0}$ and $\mathcal{L}$ are recoverable from $T$.

4.1 Review of $\omega$-completeness and finitary consistency for fragments Let $\mathcal{L}^{\prime}$ be a countable fragment of $\mathcal{L}_{\omega_{1}, \omega}$ and $T^{\prime} \subseteq \mathcal{L}^{\prime}$ a set of sentences. $T^{\prime}$ is $\omega$-complete in $\mathcal{L}^{\prime}$ if and only if (1) and (2) hold.

1. For every sentence $\mathcal{F} \in \mathscr{L}^{\prime}$, either $\mathcal{F} \in T^{\prime}$ or $(\neg \mathcal{F}) \in T^{\prime}$.

2. For any sentence $\left(\vee_{i} \mathcal{F}_{i}\right) \in T^{\prime}$, there is an $i$ such that $\mathcal{F}_{i} \in T^{\prime}$.

Say $T^{\prime}$ is finitarily consistent if and only if no contradiction can be derived from $T^{\prime}$ using only the finitary rules of $\mathscr{L}_{\omega_{1}, \omega}$. The infinitary step being avoided is deriving an infinite conjunction by deriving each of its components. Say $T^{\prime}$ is $\omega$-consistent if and only if for any sentence $\left(\vee_{i} \mathcal{F}_{i}\right) \in \mathscr{L}^{\prime}$, if $T^{\prime} \cup\left\{\vee_{i} \mathcal{F}_{i}\right\}$ is finitarily consistent, then there is an $i$ such that $T^{\prime} \cup\left\{\mathcal{F}_{i}\right\}$ is finitarily consistent.

Proposition 4.1 If $T^{\prime}$ is finitarily consistent and $\omega$-complete, then $T^{\prime}$ has a model.

Proof Note that $T^{\prime}$ is $\omega$-consistent. The model is constructed by extending $T^{\prime}$ to a finitarily consistent and $\omega$-complete set of sentences that includes Henkin axioms. At each stage of the construction, the set of sentences up to that point is $\omega$-consistent.

Proposition 4.2 Suppose for all $\beta \leq \gamma<\lambda, T_{\beta}$ is finitarily consistent and $\omega$ complete in the fragment $\mathscr{L}_{\beta}, T_{\beta} \subseteq T_{\gamma}$, and $\mathscr{L}_{\beta} \subseteq \mathcal{L}_{\gamma}$. Then $\cup\left\{T_{\beta} \mid \beta<\lambda\right\}$ is finitarily consistent and $\omega$-complete in the fragment $\cup\left\{\mathcal{L}_{\beta} \mid \beta<\lambda\right\}$.

Morley [13] showed that the scatteredness of $T$ implies the countable models of $T$ can be arranged in a hierarchy of height at most $\omega_{1}$ based on Scott rank with at most countably many models on each level. The current section revisits [15] and presents a $\Sigma_{1}$ enumeration of the countable models of $T$ with a recursion-theoretic eye on some constructive details. The enumeration is a continuous tree $\mathcal{T} \mathcal{R}(\mathcal{T})$ with at most $\omega_{1}$ levels and at most countably many nodes on each level. Each node is a theory $T^{\prime}$ finitarily consistent and $\omega$-complete in a fragment $\mathscr{L}_{T^{\prime}}$ with $T \subseteq T^{\prime}$ and $\mathscr{L}_{0} \subseteq \mathscr{L}_{T^{\prime}}$. Each $T^{\prime}$ has an atomic model, and the class of all such models is the class of all countable models of $T$.

The enumeration of $\mathcal{T} \mathcal{R}(T)$ is as follows. 
Level 0 Call $T^{\prime}$ a node if and only if $T^{\prime}$ is a finitarily consistent and $\omega$-complete extension of $T$ in the fragment $\mathscr{L}_{0}\left(=\mathscr{L}_{T^{\prime}}\right)$.

Level $\lambda$ (limit) Call $T^{\prime}$ a node if and only if there is a sequence $T_{\beta}(\beta<\lambda)$ such that $T_{\beta}$ is on level $\beta, T_{\beta} \subseteq T_{\gamma}(\beta<\gamma<\lambda)$, and $T^{\prime}=\cup\left\{T_{\beta} \mid \beta<\lambda\right\}$. $\mathcal{L}_{T^{\prime}}=\cup\left\{\mathcal{L}_{T_{\beta}} \mid \beta<\lambda\right\}$.

Level $\delta+1$ Suppose $S$ is a node on level $\delta$, that is, a finitarily consistent theory $\omega$-complete in its fragment $\mathcal{L}_{S}$. If $S$ is $\omega$-categorical, then $S$ has no successors on level $\delta+1$. Otherwise, $S$ has a nonprincipal $n$-type $p(\vec{x})$. Let $\mathscr{L}_{S}^{\prime}$ be the least fragment of $\mathscr{L}_{\omega_{1}, \omega}$ extending $\mathscr{L}_{S}$ and containing the conjunction

$$
\wedge\{\mathcal{F}(\vec{x}) \mid \mathcal{F}(\vec{x}) \in p(\vec{x})\}
$$

for every nonprincipal $n$-type $p(\vec{x})$ of $S$ for all $n>0$. Say $T^{\prime}$ is a successor of $S$ on level $\delta+1$ if $T^{\prime}$ is a finitarily consistent and $\omega$-complete extension of $S$ in the fragment $\mathscr{L}_{S}^{\prime}\left(=\mathscr{L}_{T^{\prime}}\right)$.

Proposition 4.3 If $\beta<\omega_{1}$, then $\mathcal{T} \mathcal{R}(T)$ has only countably many nodes on level $\beta$.

Proof By induction on $\beta$. Level 0 is countable by clause (b) of the definition of scattered. Suppose $S$ is on level $\delta$. Assume $\mathscr{L}_{S}$ is countable. The set of all nonprincipal $n$-types of $S$ is countable by clause (a) of the definition of scattered; hence, $\mathcal{L}_{S}^{\prime}$ is countable. The set of all successors of $S$ on level $\delta+1$ is countable by clause (b) of the definition of scattered.

Let $T^{\prime}$ be any node on the countable limit level $\lambda$. Let $\mathscr{L}_{\lambda}$ be the least fragment extending all the $\mathscr{L}_{S}$ s for all theories $S$ on all levels below $\lambda$. By induction, $\mathscr{L}_{\lambda}$ is countable. Let $T^{\prime \prime}$ be any finitarily consistent and $\omega$-complete extension of $T^{\prime}$ in $\mathcal{L}_{\lambda}$. The set of all $T^{\prime \prime} \mathrm{s}$ is countable, so the set of all $T^{\prime} \mathrm{s}$ is countable.

Let $\mathcal{T} \mathcal{R}(T)\lceil\beta$ be the restriction of $\mathcal{T} \mathcal{R}(T)$ to the levels below $\beta$.

\section{Proposition 4.4}

(i) If $\beta<\alpha<\omega_{1}$ and $L(\alpha, T)$ is $\Sigma_{1}$ admissible, then

$$
(\mathcal{T} \mathcal{R}(T)\lceil\beta) \in L(\alpha, T) .
$$

(ii) There exists a lightface $\Sigma_{1}^{Z F}$ formula $g(u, v, w)$ such that for all scattered $T$, all countable $\Sigma_{1}$ admissible $L(\alpha, T)$, and all $b \in L(\alpha, T)$,

$$
(\mathcal{T} \mathcal{R}(T)\lceil\beta)=b \Longleftrightarrow L(\alpha, T) \models g(T, \beta, b) .
$$

Proof By a $\Sigma_{1}^{L(\alpha, T)}$ recursion that relies on Theorem 3.3. Suppose

$$
(\mathcal{T} \mathcal{R}(T)\lceil(\delta+1)) \in L(\alpha, T),
$$

and theory $S$ is on level $\delta$. The set of nonprincipal types of $S$ is the unique $s \in L(\alpha, T)$ that satisfies the $\Sigma_{1} \mathcal{F}$ of Theorem 3.3 with $p$ and $b$ both equal to $S$. The statement " $q$ is a nonprincipal type of $S$ " is lightface $\Delta_{0}^{Z F}$ and corresponds to the formula $D(x, y)$ of (3.1). The fragment $\mathcal{L}_{S}^{\prime}$ was defined just before Equation (4.1). The set of successors of $S$ on level $\delta+1$ is obtained from Theorem 3.3 with parameters $\langle p, b\rangle$ equal to $\left\langle S, \mathcal{L}_{S}^{\prime}\right\rangle$. 
Let $\mathcal{A}$ be a countable model of $T$ (a scattered theory as above). The Scott analysis of $\mathcal{A}$ differs little from its tree analysis:

$$
\begin{aligned}
& T(0, \mathcal{A})=\text { theory of } \mathcal{A} \text { in } \mathscr{L}_{0} \text {, and } \mathscr{L}_{T(0, \mathcal{A})}=\mathscr{L}_{0} \text {. } \\
& T(\lambda, \mathcal{A})=\cup\{T(\beta, \mathcal{A}) \mid \beta<\lambda\} \text {. } \\
& \mathcal{L}_{T(\lambda, \mathcal{A})}=\cup\left\{\mathcal{L}_{T(\beta, \mathcal{A})} \mid \beta<\lambda\right\} . \\
& \mathcal{L}_{T(\delta+1, \mathcal{A})}=\mathscr{L}_{T(\delta, A)}^{\prime} \text { (defined similarly to } \mathscr{L}_{S}^{\prime} \text { on level } \delta+1 \text { of } \mathcal{T} \mathcal{R}(T) \text { above). } \\
& T(\delta+1, \mathcal{A})=\text { theory of } \mathcal{A} \text { in } \mathscr{L}_{T(\delta+1, \mathcal{A})} \text {. }
\end{aligned}
$$

Recall from Section 2 the definition of $d_{\mathcal{A}}$, the distinction rank of $\mathcal{A}$, and the argument that the Scott rank of $\mathcal{A}$ is either $d_{\mathcal{A}}$ or $d_{\mathcal{A}}+1$. Clearly, there is a $\delta<\omega_{1}$ such that for all $n$, any distinction made between $n$-tuples of $\mathcal{A}$ by a formula of $\mathcal{L}_{T\left(\omega_{1}, \mathcal{A}\right)}$ is made by a formula of $\mathcal{L}_{T(\delta, \mathcal{A})}$. The tree rank of $\mathcal{A}$ is defined by

$$
\operatorname{tr}(\mathcal{A})=\text { least } \delta[\mathcal{A} \text { is the atomic model of } T(\delta, \mathcal{A})] .
$$

Proposition $4.5 \operatorname{tr}(\mathcal{A}) \leq \operatorname{sr}(\mathcal{A})$.

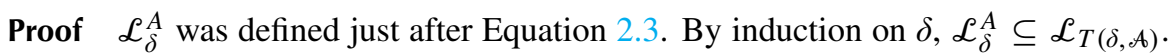
Thus $T_{\mathrm{sr}(\mathcal{A})}^{\mathcal{A}} \subseteq T(\operatorname{sr}(\mathcal{A}), \mathcal{A})$. But $\mathcal{A}$ is an atomic, hence homogeneous, model of $T_{\mathrm{sr}(\mathcal{A})}^{\mathcal{A}}$, and so $\mathcal{A}$ is an atomic model of $T(\operatorname{sr}(\mathscr{A}), \mathcal{A})$.

Proposition 4.6 Suppose $\mathcal{A} \models T$ and $\mathcal{L}(\alpha,<T, \mathcal{A}>)$ is $\Sigma_{1}$ admissible. Then

$$
\operatorname{tr}(\mathcal{A})<\alpha \longrightarrow \operatorname{sr}(\mathcal{A})<\alpha .
$$

Proof Suppose not. Then $D$, the set of all distinctions between $n$-tuples (all $n>0$ ) of $\mathcal{A}$ made by formulas of $\mathcal{L}_{T(\operatorname{tr}(\mathcal{A}), \mathcal{A})}$, belongs to $\mathcal{L}(\alpha,\langle T, \mathcal{A}\rangle)$ by Proposition 4.4. And there is an unbounded $\Sigma_{1}^{L(\alpha,\langle T, \mathcal{A}\rangle)}$ map of $D$ into $\alpha$, a violation of the $\Sigma_{1}$ admissibility of $\mathcal{L}(\alpha,\langle T, \mathcal{A}\rangle)$. The map carries each distinction $d \in D$ to the least $\delta$ such that $d$ is made by some formula of $\mathscr{L}_{\delta}^{\mathcal{A}}$.

A theory $T$ can be scattered up to a point. The tree $\mathcal{T} \mathcal{R}(T)$ is said to be scattered below $\beta$ if the notion of scattered enumeration succeeds for $T$ on all levels below $\beta$. To be more precise, $\mathcal{T} \mathcal{R}(T)$ has only countably many nodes (perhaps none) on each level below $\beta$.

Proposition 4.7 Suppose $\alpha<\omega_{1}, L(\alpha, T)$ is $\Sigma_{1}$ admissible, $T$ is scattered below $(\alpha+1)$, and $T$ has a model of Scott rank $\geq \beta$ for each $\beta<\alpha$. Then there exists $a$ theory $T_{\alpha}$ on level $\alpha$ of $\mathcal{T} \mathcal{R}(T)$ such that $T_{\alpha}$ is $\Delta_{1}^{L(\alpha, T)}$.

Proof By Proposition 4.6, $\mathcal{T} \mathcal{R}(T)$ has nodes on all levels below $\alpha$ if an $\mathcal{A}$ can be found that satisfies the hypotheses of Proposition 4.6 and also $\operatorname{sr}(\mathcal{A}) \geq \alpha$. To find $\mathcal{A}$ through Barwise Compactness, consider the following set $Z$ of sentences.

(Z1) Introduce a constant $\underline{e}$ to name each $e \in L(\alpha, T)$. Add the atomic diagram (in the sense of $\left.\mathcal{L}_{\omega_{1}, \omega}\right)$ of $L(\alpha, T)$ to $Z$. For each $\beta<\alpha$,

$$
\forall x[x \in \underline{\beta} \longleftrightarrow \vee\{x=\underline{\gamma} \mid \gamma<\beta\}]
$$

is a typical member of $(Z 1)$. Any model of $(Z 1)$ is an end extension of $L(\alpha, T)$.

(Z2) Introduce a new constant $\underline{d}$ and add sentences saying $\underline{d}$ is an ordinal greater than $\underline{\beta}$ for each $\beta<\alpha$. 
(Z3) $\quad$ Add $\mathcal{A} \models T$ and $\operatorname{sr}(\mathcal{A})>\beta$ for each $\beta<\alpha$.

(Z4) Add the axioms for $\Sigma_{1}$ admissibility.

Let $M$ be a model of $Z$ that omits $\alpha$ but extends $L(\alpha, T)$ as in [5] or [7]. $L(\alpha,\langle T, \mathcal{A}\rangle)$ is $\Sigma_{1}$ admissible; otherwise $\alpha \in M$. (Z3) insures $\operatorname{sr}(\mathcal{A}) \geq \alpha$.

Let $T^{\prime}$ denote an arbitrary node below level $\alpha$. Call $T^{\prime}$ unbounded if $T^{\prime}$ has extensions to theories on arbitrarily high levels below $\alpha$. Then $T$ can be regarded as an unbounded node.

Suppose $T^{\prime}$ is an unbounded node below level $\beta$ for some $\beta<\alpha$; then $T^{\prime}$ has an unbounded extension on level $\beta$. Otherwise, the $\Sigma_{1}$ admissibility of $L(\alpha, T)$ implies $T^{\prime}$ is bounded.

There exists a $\beta_{0}<\alpha$ and an unbounded node $T_{\beta_{0}}$ on level $\beta_{0}$ such that for all $\beta \in\left(\beta_{0}, \alpha\right), T_{\beta_{0}}$ has a unique unbounded extension on level $\beta$. Otherwise, a tree $\mathcal{U}$ of unbounded nodes can be constructed such that $\mathcal{U}$ is isomorphic to the binary branching tree $2^{<\omega}$, and the branches of $U$ define a continuum of nodes on some level $\alpha_{0} \leq \alpha$ of $\mathcal{T} \mathcal{R}(T)\lceil(\alpha+1)$.

The set $S_{u b}$ of unbounded nodes above $T_{\beta_{0}}$ form an expanding sequence whose union is the desired $T_{\alpha}$. To see $S_{u b}$ is $\Delta_{1}^{L(\alpha, T)}$, let $N_{\gamma}$ be the set of all nodes on level $\gamma$ extending $T_{\beta_{0}}$ for each $\gamma \in\left(\beta_{0}, \alpha\right)$. Then $N_{\gamma}$, as a function of $\gamma$, is $\Sigma_{1}^{L(\alpha, T)}$ by Proposition 4.4, and $\left(N_{\gamma}-S_{u b}\right) \in L(\alpha, T)$ since $N_{\gamma} \cap S_{u b}$ has just one element. There is a $\Sigma_{1}^{L(\alpha, T)}$ function that takes each node $e \in\left(N_{\gamma}-S_{u b}\right)$ to a bound on the levels occupied by extensions of $e$. But then there is a strict upper bound $b<\alpha$ on the levels occupied by extensions of members of $\left(N_{\gamma}-S_{u b}\right)$. Any such $b$ singles out the unique member of $N_{\gamma} \cap S_{u b}$.

Proposition 4.8 Suppose $\alpha \leq \omega_{1}, L(\alpha, T)$ is $\Sigma_{2}$ admissible, $T$ is scattered below $\alpha$, and $T$ has models of arbitrarily high Scott rank less than $\alpha$. Then there exists a theory $T_{\alpha}$ on level $\alpha$ of $\mathcal{T} \mathcal{R}(T)$ such that $T_{\alpha}$ is $\Delta_{1}^{L(\alpha, T)}$.

Proof The proof is similar to that of Proposition 4.7. The only difference is in the handling of $U$. Then and now $U$ can be defined by a $\Sigma_{2}^{L(\alpha, T)}$ recursion of length $\omega$, since the set of unbounded nodes is $\Pi_{1}^{L(\alpha, T)}$. But now the $\Sigma_{2}$ admissibility of $L(\alpha, T)$ implies $U \in L(\alpha, T)$, and so the branches of $U$ define a continuum of nodes on some level $\alpha_{0}<\alpha$ of $\mathcal{T} \mathcal{R}(T)$.

Two $\mathcal{L}$-structures are said to be $\mathcal{L}_{\omega_{1}, \omega}$-equivalent if they satisfy the same sentences of $\mathcal{L}_{\omega_{1}, \omega}$. (Recall that if $\mathcal{A}$ is countable and $\mathscr{L}_{\omega_{1}, \omega}$-equivalent to $\mathcal{B}$, then $\mathcal{A}$ is $\mathscr{L}_{\infty, \omega^{-}}$ equivalent to $\mathcal{B}$.)

Theorem 4.9 Suppose Vaught's Conjecture fails for $T$. Then there exist $T_{\beta}, \mathcal{A}_{\beta}$, and $\mathscr{L}_{\beta}\left(\beta \leq \omega_{1}\right)$ such that

(i) if $\beta<\omega_{1}$, then $T_{\beta}$ is an $\omega$-complete theory in the countable fragment $\mathcal{L}_{\beta}$;

(ii) if $\beta \leq \gamma \leq \omega_{1}$, then $T_{\beta} \subseteq T_{\gamma}, \mathcal{A}_{\beta} \subseteq \mathcal{A}_{\gamma}$, and $\mathcal{L}_{\beta} \subseteq \mathcal{L}_{\gamma}$;

(iii) if $\lambda$ (limit) $\leq \omega_{1}$, then $T_{\lambda}=\cup\left\{T_{\beta} \mid \beta<\lambda\right\}$ and $\mathcal{A}_{\lambda}=\cup\left\{\mathcal{A}_{\beta} \mid \beta<\lambda\right\}$;

(iv) $T_{\omega_{1}}$ is $\Delta_{1}^{L\left(\omega_{1}, T\right)}$ definable;

(v) if $\beta \leq \omega_{1}$, then $\mathcal{A}_{\beta}$ is an atomic model of $T_{\beta}$;

(vi) if $\beta<\omega_{1}$, then $\mathcal{A}_{\beta+1}$ realizes a nonprincipal type of $T_{\beta}$;

(vii) (Harnik and Makkai [6]) The cardinality of $\mathcal{A}_{\omega_{1}}$ is $\omega_{1}$, and $\mathcal{A}_{\omega_{1}}$ is not $\mathcal{L}_{\omega_{1}, \omega^{-}}$ equivalent to any countable model. ${ }^{3}$ 
Proof A uncountable model $\mathcal{A}_{\omega_{1}}$ of $T$ is constructed so that it is not $\mathscr{L}_{\omega_{1}, \omega^{-}}$ equivalent to any countable model. By Proposition 4.8, there is a theory $T_{\omega_{1}}$ on level $\omega_{1}$ of $\mathcal{T} \mathcal{R}\left(\omega_{1}\right)$ such that $T_{\omega_{1}}$ is $\Delta_{1}^{L\left(\omega_{1}, T\right)}$. Thus $T_{\omega_{1}}=\cup\left\{T_{\gamma} \mid \gamma<\omega_{1}\right\}$, and $(\gamma \leq \delta) \rightarrow\left(T_{\gamma} \subseteq T_{\delta}\right) . p$, the parameter used in the $\Delta_{1}^{L(\alpha, T)}$ definition of $T_{\omega_{1}}$, belongs to $L\left(\alpha_{0}, T\right)$ for some $\alpha_{0}<\omega_{1}$. Define

$$
K=\left\{\beta \mid \alpha_{0}<\beta<\omega_{1} \wedge L(\beta, T) \preccurlyeq 1 L\left(\omega_{1}, T\right)\right\} .
$$

(Recall that $X \preccurlyeq 1 Y$ means $X$ is a $\Sigma_{1}^{Z F}$ substructure of $Y$.) Let $\left\{\gamma_{\delta} \mid \delta<\omega_{1}\right\}$ be an increasing enumeration of $K$. Then $L\left(\gamma_{\delta}, T\right)$ is $\Sigma_{1}$ admissible, and so

$$
T_{\gamma_{\delta}}=T_{\omega_{1}} \cap L\left(\gamma_{\delta}, T\right)
$$

by Proposition 4.4(i). Also $T_{\gamma_{\delta}}$ is $\Delta_{1}^{L\left(\gamma_{\delta}, T\right)}$ definable via the same $\Delta_{1}$ definition that works for $T_{\omega_{1}}$, since $p \in L\left(\gamma_{\delta}, T\right) \preccurlyeq 1 L\left(\omega_{1}, T\right)$.

Structures $\mathcal{A}_{\delta}\left(\delta \leq \omega_{1}\right)$ and inclusion maps $i_{\beta, \delta}: \mathcal{A}_{\beta} \longrightarrow \mathcal{A}_{\delta}(\beta<\delta)$ are defined by recursion on $\delta$. The map $i_{\beta, \delta}$ will be elementary with respect to the language $\mathcal{L}_{\gamma \beta}$; that is, any sentence of $\mathcal{L}_{\gamma_{\beta}}$ with parameters in $\mathcal{A}_{\beta}$ and true in $\mathcal{A}_{\beta}$ will also be true in $\mathcal{A}_{\delta}$.

Stage 0 Structure $\mathcal{A}_{0}$ is the countable atomic model of $T_{\gamma_{0}}$.

Stage $\delta+1 \quad$ Assume $\mathcal{A}_{\delta}$ is the countable atomic model of $T_{\gamma_{\delta}}$. Extend $\mathcal{A}_{\delta}$ to $\mathcal{A}_{\delta+1}$, the countable atomic model of $T_{\gamma_{\delta+1}}$, so that the inclusion map $i_{\delta, \delta+1}$ is $\mathcal{L}_{\gamma_{\delta^{-}}}$ elementary.

Stage $\lambda$ (limit $\leq \omega_{1}$ ) Let

$$
\mathcal{A}_{\lambda}=\cup\left\{\mathcal{A}_{\delta} \mid \delta<\lambda\right\} .
$$

For all $\delta<\delta^{\prime}<\lambda$, assume the inclusion map $i_{\delta, \delta^{\prime}}$ is $\mathscr{L}_{\gamma_{\delta}}$-elementary. Then for each $\delta<\lambda, \mathcal{A}_{\lambda}$ is an $\mathscr{L}_{\gamma_{\delta}}$-elementary extension of $\mathcal{A}_{\delta}$ and so is a model of $T_{\gamma_{\delta}}$. Thus $\mathcal{A}_{\lambda}$ is a model of $T \gamma_{\lambda}$.

To see that $\mathcal{A}_{\lambda}$ is an atomic model of $T \gamma_{\lambda}$, let $\vec{a}$ be an $n$-tuple of $\mathcal{A}_{\lambda}$. For some $\delta<\lambda, \vec{a}$ is an $n$-tuple of $\mathcal{A}_{\delta}$ and realizes some atom $\mathcal{F}(\vec{x})$ of $T_{\gamma_{\delta}}$. Then $\mathcal{F}(\vec{x})$ is an atom of $T_{\lambda}$, because $L\left(\gamma_{\delta}, T\right) \preccurlyeq 1 L(\lambda, T)$. Hence, $\vec{a}$ realizes $\mathcal{F}(\vec{x})$ in $\mathcal{A}_{\lambda}$, since $i_{\delta, \lambda}$ is $\mathscr{L}_{\delta}$-elementary.

If $\mathscr{A}_{\omega_{1}}$ were $\mathcal{L}_{\omega_{1}, \omega}$-equivalent to some countable model, then it would be an atomic model of $T_{\gamma_{\delta}}$ for some $\delta<\omega_{1}$. But $\mathcal{A}_{\delta+1}$, hence $\mathcal{A}_{\omega_{1}}$, realizes a nonprincipal type of $T_{\gamma_{\delta}}$.

\section{Absoluteness of Vaught's Conjecture}

Let $V C(T)$ be the predicate 'Vaught's conjecture holds for $T$ '. Morley's work [13] implies that $V C(T)$ is absolute. The enumeration tree, $\mathcal{T} \mathcal{R}(T)$, of Section 4 is applied below to make the statement of $V C(T)$ more precise and to see in some detail how $T$ can satisfy Vaught's Conjecture. Suppose an attempt is made to develop $\mathcal{T} \mathcal{R}(T)$ and the attempt fails to produce a tree with only countably many nodes on each level and $\omega_{1}$ many nonempty levels. Then there must be a countable $\beta$ such that one of the following holds:

(1) $\beta=0$ and $T$ has uncountably many finitarily consistent, $\omega$-complete extensions in $\mathscr{L}_{0}$; 
(2) $\beta=\delta+1$, some theory $S$ is on level $\delta$, and for some $n$, the set of $n$-types of $S$ is uncountable;

(3) $\beta=\delta+1$, some theory $S$ is on level $\delta$, for all $n$ the set of $n$-types of $S$ is countable, and the set of all finitarily consistent, $\omega$-complete extensions of $S$ in $\mathscr{L}_{S}^{\prime}$ is uncountable. $\mathcal{L}_{S}^{\prime}$ is defined just before (4.1).

(4) $\beta=\lambda$ and the set of nodes on level $\lambda$ is uncountable.

(5) level $\beta$ is empty.

Define the Vaught Rank of $T, \operatorname{vr}(T)$ to be the least countable $\beta$ that satisfies one of (1) - (5) above. (If there is no such $\beta$, let $\operatorname{vr}(T)$ be $\omega_{1}$.) Define the predicate $V C(T)$ by $\operatorname{vr}(T)<\omega_{1}$. Suppose $\operatorname{vr}(T)=\beta<\omega_{1}$. If $\beta=0$, then $T$ has $2^{\omega}$ finitarily consistent, $\omega$-complete extensions in $\mathscr{L}_{0}$ by Theorem 3.1 , hence, $2^{\omega}$ many countable models. The same holds in cases (3) and (4). If (5) holds, then $T$ has only countably many countable models, and each one is the atomic model of a theory on some level of $\mathcal{T} \mathcal{R}(T)$ below level $\beta$. Suppose case (2) holds. Then for some $n$, there are $2^{\omega} n$ types of $S$ by Theorem 3.1, hence, $2^{\omega}$ many countable models of $T$.

Recall that

$$
\omega_{1}^{L(T)}=\text { least } \gamma[L(T) \models(\gamma \text { is uncountable })] .
$$

Proposition 5.1 The predicate, Vaught's Conjecture holds for T, is

$$
\Sigma_{1}^{L\left(\omega_{1}^{L(T)}, T\right)} \text {, hence } \Sigma_{2}^{1} \text {. }
$$

Proof By Proposition 4.4, $\mathcal{T} R(T) \subseteq L\left(\omega_{1}, T\right)$ and is $\Sigma_{1}^{L\left(\omega_{1}, T\right)}$. The statement $V C(T)$ says "at some level $\gamma<\omega_{1}$, either (a) $\mathcal{T} \mathcal{R}(T)$ ends or (b) blows up; that is, a perfect kernel of theories or types is manifest." Let $\alpha_{0}$ be the least $\alpha>\gamma$ such that $L(\alpha, T)$ is $\Sigma_{1}$ admissible.

Suppose (a) holds. Then Levy-Shoenfield Absoluteness implies $\alpha_{0}<\omega_{1}^{L(T)}$, and there is an $\mathcal{L}_{\omega_{1}, \omega}$ sentence $\mathcal{K} \in L\left(\alpha_{0}, T\right)$ that expresses the fact that every model of $T$ is an atomic model of some theory on some level at or below $\gamma$ of $\mathcal{T} \mathcal{R}(T)$.

Suppose (b) holds. Theorem 3.1 implies the existence of a perfect kernel of theories or types. A coding of some such perfect kernel by a real is constructible from any counting of $\alpha_{0}$. The proof of Theorem 3.1 relies on the consistency of a certain set $Z$ of axioms. $Z$ is $\Sigma_{1}^{L\left(\alpha_{0}, T\right)}$, and the consistency of $Z$ is $\Pi_{1}^{L\left(\alpha_{0}, T\right)}$. Hence LevyShoenfield Absoluteness implies $\alpha_{0}<\omega_{1}^{L(T)}$, and so a code for the perfect kernel belongs to $L\left(\omega_{1}^{L(T)}, T\right)$.

Proposition 5.2 Suppose T is a counterexample to Vaught's Conjecture. Then there is a theory $T_{\omega_{1}}$ on level $\omega_{1}$ of $\mathcal{T} \mathcal{R}(T)$ such that $T_{\omega_{1}}$ is $\Delta_{1}^{L\left(\omega_{1}, T\right)}$. For all countable $\beta$, $T_{\beta}$, the restriction of $T_{\omega_{1}}$ to level $\beta$, has an atomic model whose Scott rank is $\beta$.

Proof By Proposition 4.8.

Suppose $L(\alpha, T)$ is $\Sigma_{1}$ admissible, $\mathcal{A}$ is a countable model of $T$, and $\omega_{1}^{\text {A }}=\alpha$. According to (2.6), $\mathcal{A}$ is a homogenous model of $T_{\alpha}^{\mathcal{A}} ; \mathcal{A}$ is said to be $\alpha$-saturated if every $n$-type $(n \geq 1)$ of $T_{\alpha}^{\mathcal{A}}$ is realized in $\mathcal{A}$.

Theorem 5.3 Suppose $T$ is a counterexample to Vaught's Conjecture. Then there is a $\Delta_{1}^{L\left(\omega_{1}, T\right)}$ theory $T_{\omega_{1}}$ on level $\omega_{1}$ of $\mathcal{T} \mathcal{R}(T)$ and a closed unbounded set $C \subseteq \omega_{1}$ such that $\forall \alpha \in C: T_{\alpha}$, the restriction of $T_{\omega_{1}}$ to level $\alpha$, has an atomic model $\mathcal{A}_{\alpha}$ of 
Scott rank $\alpha$ and an $\alpha$-saturated model $\mathscr{B}_{\alpha}$ of Scott rank $\alpha+1$. The atomic models form an expanding chain and each inclusion $\mathcal{A}_{\beta} \subset \mathcal{A}_{\gamma}(\beta<\gamma)$ is elementary with respect to the language of $T_{\beta}$.

Proof Proposition 4.8 provides $T_{\omega_{1}}$. Let $p \in L\left(\omega_{1}, T\right)$ be the parameter needed for the $\Delta_{1}^{L\left(\omega_{1}, T\right)}$ definition of $T_{\omega_{1}}$. For any $\alpha$, let $\alpha^{+}$be the least $\beta>\alpha$ such that $L(\beta, T)$ is $\Sigma_{1}$ admissible.

For $x \in L(T)$, let $H_{1}(x)$ be the $\Sigma_{1}$ hull of $x$ in $L(T)$. Recall that

$$
x \subseteq H_{1}(x) \preccurlyeq 1 L(T)
$$

and that $x$ and $H_{1}(x)$ have the same cardinality in $L(T)$. An expanding sequence of countable $\Sigma_{1}$ hulls, $H^{\delta}\left(\delta<\omega_{1}\right)$, is defined by recursion on $\delta$.

$H^{0}$ is $H_{1}\left(\left\{\operatorname{tc}(p), \omega_{1}, \operatorname{tc}(T)\right\}\right)$. (tc is transitive closure.) Note that $\omega_{1}^{+}, \omega \in H^{0}$; if $d<e<\omega_{1}$ and $e \in H^{0}$, then $d \in H^{0}$. Let $c_{0}$ be the lub of the countable ordinals in $H^{0}$. Let $L\left(\beta_{0}, T\right)$ be the transitive collapse of $H^{0}$. Then

$$
c_{0}=\omega_{1}^{L\left(\beta_{0}, T\right)} \text { and } L\left(c_{0}^{+}, T\right) \subseteq L\left(\beta_{0}, T\right) .
$$

Stage $\delta+1 \quad$ Assume $H^{\delta}$ is countable in $V$. Then $H^{\delta} \cap \omega_{1}$ is a proper initial segment of $\omega_{1}$. Let $c_{\delta}$ be the least countable ordinal not in $H^{\delta}$. $H^{\delta+1}$ is $H_{1}\left(H^{\delta} \cup\left\{c_{\delta}\right\}\right)$.

Stage $\lambda$ (limit) Let $H^{\lambda}$ be $\cup\left\{H_{\delta} \mid \delta<\lambda\right\}$. Then $C=\left\{c_{\delta} \mid \delta<\omega_{1}\right\}$ is a closed unbounded set. Let $L\left(\beta_{\delta}, T\right)$ be the transitive collapse of $H^{\delta}$. Then

$$
c_{\delta}=\omega_{1}^{L\left(\beta_{\delta}, T\right)} \text { and } L\left(c_{\delta}^{+}, T\right) \subseteq L\left(\beta_{\delta}, T\right) .
$$

Let $T_{c_{\delta}}$ be the restriction of $T_{\omega_{1}}$ to level $c_{\delta}$ of $\mathcal{T} \mathcal{R}(T)$. Then $T_{c_{\delta}}$ is $\Delta_{1}^{L\left(c_{\delta}, T\right)}$ via parameter $p$, and $N$, the set of nonprincipal types of $T_{c_{\delta}}$, is nonempty and countable in $V$. Then $T_{c_{\delta}} \in L\left(c_{\delta}^{+}, T\right)$, and so $N \in L\left(c_{\delta}^{+}, T\right)$ by Theorem 3.1. Hence the structure $L\left[c_{\delta}, T ; T_{c_{\delta}}, N\right]$ (i.e., $L\left(c_{\delta}, T\right)$ with $x \in T_{c_{\delta}}$ and $x \in N$ as additional atomic predicates) is $\Sigma_{1}$ admissible because no subset of $c_{\delta}$ in $L\left(\beta_{\delta}, T\right)$ can define a counting of $\omega_{1}^{L\left(\beta_{\delta}, T\right)}$. Now the construction of $M$ in the proof of Theorem 6.1 can be imitated to produce a model $\mathscr{B}$ of $T_{c_{\delta}}$ such that $\mathscr{B}$ realizes all the types in $N$ and $\omega_{1}^{\mathscr{B}}=c_{\delta}$. The atomic $\mathcal{A}_{\beta}$ s are supplied by Theorem 4.9.

\section{Bounds on Scattered Theories}

Once again $\mathscr{L}$ is a countable first-order language, $\mathscr{L}_{0}$ is a countable fragment of $\mathscr{L}_{\omega_{1}, \omega}$, and $T \subseteq \mathscr{L}_{0}$ has a model. $\mathscr{L}$ and $\mathscr{L}_{0}$ are effectively recoverable from $T_{0} . T$ is scattered below $\beta$ as was defined just before Proposition 4.7.

Theorem 6.1 Suppose $\alpha<\omega_{1}, L(\alpha, T)$ is $\Sigma_{2}$ admissible, $T$ is scattered below $\alpha$, and for each $\beta<\alpha, T$ has a model of Scott rank $\geqslant \beta$. Then $T$ has a model $\mathcal{A}$ such that $\omega_{1}^{\mathcal{A}}=\alpha$ and $\operatorname{sr}(\mathcal{A})=\alpha+1$.

Proof By Proposition 4.8, $\mathcal{T} \mathcal{R}(A)$ has a theory $T_{\alpha}$ on level $\alpha$ such that $T_{\alpha}$ is $\Delta_{1}^{\alpha}$ and $T_{\alpha}$ is $\cup\left\{T_{\beta} \mid \beta<\alpha\right\}$, where $T_{\beta}$ is a node on level $\beta$. Let $Z$ be the following set of sentences.

(Z1) The atomic diagram of $L(\alpha, T)$ in the sense of $\mathcal{L}_{\omega_{1}, \omega}$.

(Z2) Add $(\underline{d}>\underline{\beta})$ for all $\beta<\alpha . \underline{d}$ is a constant not occurring in (Z1). 
(Z3) Let $T_{\underline{d}}$ be a theory on level $\underline{d}$ of $\mathcal{T} \mathcal{R}(T)$. Add $\mathcal{A}$ is the countable atomic model of $T_{\underline{d}}$ and $\mathcal{F} \in T_{\underline{d}}$ for each sentence $\mathcal{F} \in T_{\alpha}$.

(Z4) Add $\left(b(\vec{x})\right.$ is an atom of $\left.T_{\underline{d}}\right)$ for each $b(\vec{x})$ that is an atom of $T_{\alpha}$; that is, $b(\vec{x})$ generates a principal type of $T_{\alpha}$.

(Z5) Add the axioms of $\Sigma_{1}$ admissibility. The set $Z$ is $\Sigma_{2}^{L(\alpha, T)}$, since the set of atoms of $T_{\alpha}$ is $\Pi_{1}^{L(\alpha, T)}$.

Suppose $\beta<\alpha, L(\beta, T)$ is $\Sigma_{1}$ admissible, and $Z_{\beta}$ is $Z \cap L(\beta, T)$. A fundamental fact of forcing in the setting of set theory is the Levy collapse of a cardinal to $V$ preserves replacement; furthermore, the preservation of $\Sigma_{n}$ replacement needs only $\Sigma_{n}$ replacement in $V$. To check the consistency of $Z_{\beta}$, augment $L(\alpha, T)$ by adding a generic counting of $L(\beta, T)$ to $L(\alpha, T)$ that preserves the $\Sigma_{2}$ admissibility of $L(\alpha, T)$. The set $Z_{\beta}$ can be modeled by the augmented $L(\alpha, T)$. By Proposition 4.4, $T_{\beta} \subseteq L(\beta, T)$. Interpret $\underline{d}$ as $\beta$. Interpret $\mathcal{A}$ as the atomic model of $T_{\beta}$. Such an $\mathcal{A}$ belongs to the augmented $L(\alpha, T)$ because there $T_{\beta}$ is countable. If $b(\vec{x})$ is an atom of $T_{\alpha}$ and belongs to $L(\beta, T)$, then $b(\vec{x})$ is an atom of $T_{\beta}$.

The set $Z$ has a model $M$ that is a proper end extension of $L(\alpha, T)$ but omits $\alpha$. $\omega_{1}^{\mathcal{A}} \leq \alpha$; otherwise, $\alpha$ is recursive in $\mathcal{A}$, and then $\alpha \in M$. By design $\mathcal{A} \models T_{\beta}$ for all $\beta<\alpha$; hence, $\operatorname{sr}(\mathcal{A}) \geq \alpha$ by Proposition 4.5 , and so $\omega_{1}^{\mathcal{A}}=\alpha$ by (2.6).

Suppose $\operatorname{sr}(\mathcal{A})=\alpha$. Then $\alpha \in M$ as follows. By supposition $\mathcal{A}$ is the atomic model of $T_{\alpha}$. The rank of an atom $b(\vec{x})$ of $T_{\alpha}$ is the least $\beta<\alpha$ such that $b(\vec{x})$ is an atom of $T_{\beta}$. Let $f$ be the function that carries each $\vec{a} \in \mathcal{A}$ to the rank of an atom of $T_{\alpha}$ that generates the principal type realized by $\vec{a}$ in $\mathcal{A}$. Thanks to (Z4) $f$ is definable from $T_{d}$, and so $f \in M$. Then lub(range $f$ ) $=\alpha \in M$.

Corollary 6.2 ([15]) Suppose for every countable model $\mathcal{A}$ of $T$, the Scott rank of $\mathcal{A}$ is less than or equal to $\omega_{1}^{\text {st }}$. Then Vaught's Conjecture holds for $T$.

Proof Suppose $\operatorname{VC}(T)$ fails. Then $T$ is scattered below $\omega_{1}$, and $\mathcal{T} \mathcal{R}(T)$ has nodes on every countable level. Choose an $\alpha<\omega_{1}$ such that $L(\alpha, T)$ is $\Sigma_{2}$ admissible. Then $T$ has a countable model $\mathcal{A}$ such that $\omega_{1}^{\mathscr{A}}=\alpha$ and $\operatorname{sr}(\mathcal{A})=\alpha+1$.

A more effective version of Corollary 6.2 is as follows. Define

$$
\sigma_{2}^{T}=\text { least } \alpha\left[L(\alpha, T) \text { is } \Sigma_{2} \text { admissible }\right] .
$$

$\operatorname{vr}(T)$, the Vaught rank of $T$, was defined at the beginning of Section 6.

Corollary 6.3 Suppose $T$ does not have a countable model $\mathcal{A}$ such that

$$
\omega_{1}^{\mathcal{A}}=\sigma_{2}^{T} \text { and } \operatorname{sr}(\mathcal{A})=\sigma_{2}^{T}+1 .
$$

Then $\operatorname{vr}(T)<\sigma_{2}^{T}$.

Proof If $\operatorname{vr}(T) \geqslant \sigma_{2}^{T}$, then $T$ is scattered below $\sigma_{2}^{T}$ and $\mathcal{T} \mathcal{R}(T)$ has nodes on every level below $\sigma_{2}^{T}$.

As a warmup to the main bounding results of the paper (Section 8), the above is recast as an effective bounding theorem.

Corollary 6.4 Suppose $T$ is scattered and

$$
\operatorname{sr}(\mathcal{A}) \leq \omega_{1}^{\mathcal{A}} \text { for every countable } \mathcal{A} \models T .
$$


Then $\exists \beta<\sigma_{2}^{T}$ such that

$$
\operatorname{sr}(\mathcal{A})<\beta \text { for every } \mathcal{A} \models T .
$$

Let $S A(T)$ say for every countable model $\mathscr{A}$ of $T$, the theory $T_{\omega_{1}^{A}}^{\mathcal{A}}$ is $\omega$-categorical. Steel [18], as reported in Makkai [11], showed that $V C(T)$ follows from $S A(T)$. Theorem 6.5 is an effective version of Steel's result.

The set $L(\alpha, T)$ is said to be recursively Mahlo if $L(\alpha, T)$ is $\Sigma_{1}$ admissible and every $\Delta_{1}^{L(\alpha, T)}$ closed unbounded subset of $\alpha$ has a member $\beta$ such that $L(\beta, T)$ is $\Sigma_{1}$ admissible. Define

$$
\operatorname{rm}(T)=\text { least } \gamma[L(\gamma, T) \text { is recursively Mahlo }] .
$$

Note that $\operatorname{rm}(T)<\sigma_{2}^{T}$.

Theorem 6.5 Suppose $T$ is scattered and

$$
T_{\omega_{1}^{A}}^{\mathcal{A}} \text { is } \omega \text {-categorical for every countable } \mathcal{A} \models T \text {. }
$$

Then $\exists \beta<\operatorname{rm}(T)$ such that

$$
\operatorname{sr}(\mathcal{A})<\beta \text { for every countable } \mathcal{A}=T .
$$

Proof Suppose there is no such $\beta$. Let $\alpha$ be $\operatorname{rm}(T)$. Then Proposition 4.7 supplies a $\Delta_{1}^{L(\alpha, T)}$ theory $T_{\alpha}$ on level $\alpha$ of $\mathcal{T} \mathcal{R}(T)$. Then $T_{\alpha}=\cup\left\{T_{\beta} \mid \beta<\alpha\right\}$, and $T_{\beta}$, as a function of $\beta$, is $\Sigma_{1}^{L(\alpha, T)}$.

There is a $\Sigma_{1}^{L(\alpha, T)}$ function $f_{0}$ such that $T_{\beta} \subseteq L\left(f_{0}(\beta), T\right)$ for all $\beta<\alpha$. Iteration of $f_{0}$ leads to a $\Delta_{1}^{L(\alpha, T)}$ closed unbounded set

$$
C_{0}=\left\{\gamma \mid T_{\gamma} \subseteq L(\gamma, T)\right\} .
$$

A similar argument produces a $\Delta_{1}^{L(\alpha, T)}$ closed unbounded set $C_{1}$ such that

$$
\forall \gamma \in C_{1}\left[\left(T_{\alpha} \cap L(\gamma, T)\right) \text { is } \Delta_{1}^{L(\gamma, T)}\right] .
$$

Then there is a $\Delta_{1}^{L(\alpha, T)}$ closed unbounded set $K$ such that

$$
\forall \gamma \in K\left[T_{\gamma} \subseteq L(\gamma, T) \text { and } T_{\gamma} \text { is } \Delta_{1}^{L(\gamma, T)}\right] .
$$

Hence, for some $\gamma_{0} \in K, L\left(\gamma_{0}, T\right)$ is $\Sigma_{1}$ admissible. Consequently $T_{\gamma_{0}}$ has a model $\mathscr{B}$ such that $\omega_{1}^{\mathcal{B}}=\gamma_{0}$. But then $T_{\omega_{1}^{\mathcal{B}}}^{\mathcal{B}}$, hence $T_{\gamma_{0}}$, is $\omega$-categorical and so has no extension to a node on level $\alpha$.

\section{Iterated Classical Bounding}

In this section classical bounding (reviewed in Section 1) is translated into the language of $\Sigma_{1}$ admissible sets and revised to allow for iterated use in $\Sigma_{1}$ recursive definitions in Section 8.

Let $B(x)$ be a $\Delta_{0}^{Z F}$ formula with parameter $p_{0}$. The formula $B(x)$ is $\beta$-bounded if and only if

$$
\forall c\left[B(c) \Longleftrightarrow L\left[\beta, p_{0} ; c\right] \models B(\underline{c})\right] .
$$

The set $L\left[\beta, p_{0} ; c\right]$ is the result of iterating first-order definability with $y \in c$ as an additional atomic predicate through the ordinals less than $\beta$ starting with the transitive closure (tc) of $\left\{p_{0}\right\}$. Assume $B(x)$ is $\beta$-bounded. Define

$$
c_{\beta}=c \cap L\left[\beta, p_{0} ; c\right] .
$$


Then $B(c) \Longleftrightarrow B\left(c_{\beta}\right)$. For all $z$ let $A_{z}$ be the least $\Sigma_{1}$ admissible set with $z$ as a member; thus

$$
A_{z}=L\left(\omega_{1}^{z}, \operatorname{tc}(\{z\})\right) .
$$

Let $\mathcal{F}(u, v)$ be a $\Sigma_{1}^{Z F}$ formula with parameter $p_{1}$, and let $p$ be $\left\{p_{0}, p_{1}\right\}$. Suppose for all $c$, if $B(c)$, then there exists a unique $\delta \in A_{\left\{p, \beta, c_{\beta}\right\}}$ such that

$$
A_{\left\{p, \beta, c_{\beta}\right\}} \models \mathcal{F}\left(\underline{c_{\beta}}, \underline{\delta}\right) ;
$$

designate $\delta$ by $\delta_{p, \beta, c}$.

\section{Theorem 7.1}

(i) There exists a $\delta_{p, \beta} \in A_{\{p, \beta\}}$ such that for all $c$,

$$
B(c) \longrightarrow \delta_{p, \beta, c} \leq \delta_{p, \beta} .
$$

(ii) $\delta_{p, \beta}$ can be construed as a partial function of $p$ and $\beta$ whose restriction to any $\Sigma_{1}$ admissible $A$ has a $\Sigma_{1}^{A}$ definition uniformly in $A$; that is, one $\Sigma_{1}$ formula works for all $A$.

Proof Let $Z$ be the following $\Sigma_{1}^{A_{\{p, \beta\}}}$ set of sentences. Let $\alpha=\omega_{1}^{\{p, \beta\}}$.

(Z1) Introduce constants $\underline{c}$ and $\underline{c_{\beta}}$, and put $\underline{c_{\beta}}=\underline{c} \cap L\left[\beta, p_{0} ; \underline{c}\right]$ and $B\left(\underline{c_{\beta}}\right)$ in $Z$.

(Z2) Add constants that name the elements of

$$
L\left(\alpha, \operatorname{tc}\left(\left\{p, \beta, c_{\beta}\right\}\right)\right)
$$

and sentences of $\mathcal{L}_{\omega_{1}, \omega}$ that define each element in terms of elements of lower definability rank.

(Z3) Let $\mathcal{F}(u, v)$ be $\exists w g(u, v, w)$ for some $\Delta_{0}^{Z F}$ formula $g(u, v, w)$. Add $\neg \mathcal{G}\left(c_{\beta}, \underline{\delta}, \underline{r}\right)$ for all $\delta<\alpha$ and every $\underline{r}$ that names an element of (7.6).

(Z4) Add axioms for $\Sigma_{1}$ admissibility.

Suppose $Z$ is consistent. Assume for a moment that

$$
Z \text { is countable. }
$$

As in the proof of Proposition 4.7, $Z$ has a model $M$ that is a proper end extension of (7.6) but omits $\alpha$. Then (7.6) is $\Sigma_{1}$ admissible, and so

$$
A_{\left\{p, \beta, c_{\beta}\right\}}=L\left(\alpha, \operatorname{tc}\left(\left\{p, \beta, c_{\beta}\right\}\right)\right) .
$$

But then $A_{\left\{p, \beta, c_{\beta}\right\}} \models \neg \mathcal{F}\left(c_{\beta}, \underline{\delta}\right)$ for all $\delta<\alpha$, a contradiction since

$$
\delta_{p, \beta, c_{\beta}} \in A_{\left\{p, \beta, c_{\beta}\right\}} .
$$

Thus $Z$ is inconsistent.

To remove assumption (7.7), generically extend the universe $V$ to $V^{\prime}$ so that $Z$ is countable in $V^{\prime}$. Then $Z$ is inconsistent in $V^{\prime}$, hence in $V$, by the absoluteness of provability in the sense of $\mathcal{L}_{\infty, \omega}$.

Since $Z$ is $\Sigma_{1}^{A_{\{p, \beta\}}}$, there must be an inconsistent $W \subseteq Z$ such that

$$
W \in A_{\{p, \beta\}} .
$$

The set $W$ consists of (W1), (W2), and (W3):

(W1) (Z1) and (Z4) above.

(W2) Some $A_{0} \in A_{\{p, \beta\}}$ such that $A_{0} \subseteq$ set of sentences of (Z2). 
(W3) For some $\delta_{1}<\alpha$, the sentence $\neg \mathscr{g}\left(c_{\beta}, \underline{\delta}, \underline{r}\right)$ for all $\delta<\delta_{1}$ and every $\underline{r}$ of (Z2) that names an element of $L\left(\delta_{1}, \operatorname{tc}\left(\left\{p, \beta, c_{\beta}\right\}\right)\right)$.

Then there is a deduction $D \in A_{\{p, \beta\}}$ from (W1) and (W2) of

$$
\vee\left\{\mathcal{F}\left(\underline{c_{\beta}}, \delta\right) \mid \delta<\delta_{1}\right\} \text {. }
$$

Let $\rho_{0}$ be the least $\rho$ such that there is such a $D \in L(\rho, \operatorname{tc}(\{p, \beta\}))$; let $\delta_{\{p, \beta\}}$ be the least $\delta_{1}$ associated with any such $D \in L\left(\rho_{0}, \operatorname{tc}(\{p, \beta\})\right)$. Then

$$
\delta_{p, \beta, c} \leq \delta_{p, \beta}
$$

for any $c$ such that $B(c)$ holds. The $\Sigma_{1}^{Z F}$ formula $\mathscr{H}$ that defines $\delta_{p, \beta}$ as a partial function of $p, \beta$ uniformly owes its existence to the effective nature of deducibility in $\mathcal{L}_{\omega_{1}, \omega}$. The formula $\mathcal{H}$ singles out a deduction in $A_{\{p, \beta\}}$ that establishes the value of $\delta_{p, \beta}$ and can be formulated to succeed in every $\Sigma_{1}$ admissible $A$, because $p, \beta \in A$ implies $A_{\{p, \beta\}}$ is a $\Sigma_{1}^{A}$ definable (uniformly) subclass of $A$.

\section{Enumeration of Models under Weak Scattering}

Let $\mathscr{L}_{0}$ be a countable fragment of $\mathscr{L}_{\omega_{1}, \omega}$ for some countable first-order language $\mathscr{L}$ and $T \subseteq \mathscr{L}_{0}$ a theory with a model. Assume $T$ is weakly scattered as defined in Section 1. For convenience assume $T$ mentions all formulas of $\mathscr{L}_{0}$; thus $\mathscr{L}_{0}$ and $\mathcal{L}$ are recoverable from $T$. Since $T$ need not be scattered, there is no hope of enumerating theories in $L\left(\omega_{1}, T\right)$ whose atomic models are exactly the countable models of $T$. But some useful vestiges of the constructive features of scattering carry over to weak scattering, and $L\left(\omega_{1}, T\right)$ manages to say a great deal about the countable models of $T$.

First consider $\mathcal{R} \mathcal{H}(T)$, the raw hierarchy for the countable models of $T$. On level 0 of $\mathcal{R H}(T)$, put every $T_{0}$ such that $T \subseteq T_{0}$ and $T_{0}$ is a finitarily consistent, $\omega$-complete theory of $\mathscr{L}_{0}$. (If needed, see the beginning of Section 4 for a review.)

Suppose $T_{\delta}$ is on level $\delta$ of $\mathcal{R H}(T)$. Define

$$
\delta-= \begin{cases}\delta-1 & \text { if } \delta \text { is a successor, } \\ \delta & \text { if } \delta \text { is not a successor. }\end{cases}
$$

Let $\mathscr{L}_{0}\left(T_{0-}\right)$ be $\mathscr{L}_{0}$. Assume $T_{\delta}$ extends a unique $T_{\delta-}$ on level $\delta$ - and $\mathscr{L}_{\delta}\left(T_{\delta-}\right)$ is countable. If all $n$-types $(n \geq 1)$ of $T_{\delta}$ are principal, then $\mathscr{L}_{\delta+1}\left(T_{\delta}\right)$ is undefined and $T_{\delta}$ has no extensions on level $\delta+1$. Otherwise, let $\mathcal{L}_{\delta+1}\left(T_{\delta}\right)$ be the least fragment of $\mathcal{L}_{\omega_{1}, \omega}$ extending $\mathcal{L}_{\delta}\left(T_{\delta-}\right)$ and having as a member the conjunction

$$
\wedge\{\mathcal{F}(\vec{x}) \mid \mathcal{F}(\vec{x}) \in p(\vec{x})\}
$$

for every nonprincipal $n$-type $p(\vec{x})$ of $T_{\delta}(n \geq 1)$. Since $T$ is weakly scattered, the set $\mathcal{L}_{\delta+1}\left(T_{\delta}\right)$ is countable.

On level $\delta+1$ of $\mathcal{R H}(T)$ put every $T_{\delta+1}$ that extends $T_{\delta}$ and is a finitarily consistent, $\omega$-complete theory of $\mathcal{L}_{\delta+1}\left(T_{\delta}\right)$. Put $T_{\lambda}$ on level $\lambda$ if there is a sequence $T_{\delta}(\delta<\lambda)$ such that
(a) $T_{\delta}$ is on level $\delta$;
(b) $T_{\beta} \subseteq T_{\gamma}$ if $\beta \leq \gamma$; and
(c) $T_{\lambda}=\cup\left\{T_{\delta} \mid \delta<\lambda\right\}$. 
Define $\mathcal{L}_{\lambda}\left(T_{\lambda}\right)$ to be $\cup\left\{\mathcal{L}_{\delta}\left(T_{\delta-}\right) \mid \delta<\lambda\right\}$.

It is straightforward to verify that $\mathcal{A}$ is a countable model of $T$ if and only if $\mathcal{A}$ is the atomic model of $T_{\delta}$ for some countable $\delta$. Define the raw tree rank of $\mathcal{A}$ by

$$
\operatorname{rtr}(\mathcal{A})=(\text { least } \delta)\left[\mathcal{A} \text { is the atomic model of some } T_{\delta}\right] .
$$

Propositions 4.5 and 4.6 hold when tr is rtr. Thus

$$
\operatorname{rtr}(\mathcal{A}) \leq \operatorname{sr}(\mathcal{A})
$$

and if $L(\alpha,\langle T, \mathcal{A}\rangle)$ is $\Sigma_{1}$ admissible, then

$$
\operatorname{rtr}(\mathcal{A})<\alpha \longrightarrow \operatorname{sr}(\mathcal{A})<\alpha .
$$

What matters more is what can be expressed inside $L(\alpha, T)$ when $\alpha \leq \omega_{1}$ and $L(\alpha, T)$ is $\Sigma_{1}$ admissible. Let $A_{\delta}$ be the set of all $T_{\delta}$ s on level $\delta$ of $\mathcal{R} \mathcal{H}(T)$. The set $A_{\delta}$ will be defined by a $\beta$-bounded $\Delta_{0}^{Z F}$ formula (7.1), and its definition as such, denoted by $\left\ulcorner A_{\delta}\right\urcorner$, will belong to $L(\alpha, T)$ when $\delta<\alpha$. The fragment $\mathscr{L}_{\delta}\left(T_{\delta-}\right)$ will be constructible from $T_{\delta-}$ via an ordinal $\rho_{\delta}<\alpha$ for all $T_{\delta-} \in A_{\delta-}$. The pair $\left\ulcorner A_{\delta}\right\urcorner$ and $\rho_{\delta}$ will be defined by a simultaneous $\Sigma_{1}^{L(\alpha, T)}$ recursion uniformly in $\alpha$, that is, the same $\Sigma_{1}$ formula will work for all $\alpha \leq \omega_{1}$ such that $L(\alpha, T)$ is $\Sigma_{1}$ admissible.

Consider an arbitrary $T_{\delta}$ on level $\delta$ of $\mathcal{R H}(T)$. There exists a natural recovery process that can be applied to $T_{\delta}$ to recover the unique sequence $T_{\gamma}(\gamma<\delta)$ such that

$$
\begin{aligned}
& T_{\gamma} \text { is on level } \gamma, \\
& \gamma_{1} \leq \gamma_{2} \longrightarrow T_{\gamma_{1}} \subseteq T_{\gamma_{2}}, \text { and } \\
& T_{\lambda}=\cup\left\{T_{\gamma} \mid \gamma<\lambda\right\} \text { for all limit } \lambda \leq \delta .
\end{aligned}
$$

The recovery proceeds as follows. It begins with $T_{0}$ is $T_{\delta} \cap \mathscr{L}_{0}$. If $\gamma$ is a successor, then

$$
T_{\gamma}=T_{\delta} \cap \mathscr{L}_{\gamma}\left(T_{\gamma-}\right) .
$$

If $\gamma$ is a limit, then $T_{\gamma}=\cup\left\{T_{\beta} \mid \beta<\lambda\right\}$.

The recovery process can be used to decide whether or not an arbitrary set $c$ is a theory on level $\delta$ of $\mathcal{R H}(T)$. The answer is yes if and only if $c$ passes the following tests at all levels $\gamma \leq \delta$.

Level 0 Let $c_{0}$ be $c \cap \mathscr{L}_{0} ; c_{0}$ is an extension of $T$ and a finitarily consistent, $\omega$-complete theory of $\mathscr{L}_{0}$.

Level $\gamma+\mathbf{1} \leq \boldsymbol{\delta} \quad$ Let $\mathscr{L}_{\gamma+1}\left(c_{\gamma}\right)$ be the least fragment extending $\mathcal{L}_{\gamma}\left(c_{\gamma-}\right)$ and having as a member the conjunction

$$
\wedge\{\mathcal{F}(\vec{x}) \mid \mathcal{F}(\vec{x}) \in p(\vec{x})\}
$$

for every nonprincipal $n$-type $p(\vec{x})$ of $c_{\gamma-}$. Let $c_{\gamma+1}$ be $c \cap \mathscr{L}_{\gamma+1}\left(c_{\gamma}\right) . c_{\gamma+1}$ extends $c_{\gamma}$ and is a finitarily consistent, $\omega$-complete theory of $\mathscr{L}_{\gamma+1}\left(c_{\gamma}\right)$.

Level $\lambda$ (limit) $\leq \delta \quad$ Let $c_{\lambda}$ be $\cup\left\{c_{\gamma} \mid \gamma<\lambda\right\}$; let $\mathscr{L}_{\lambda}\left(c_{\lambda}\right)$ be $\cup\left\{\mathcal{L}_{\gamma}\left(c_{\gamma-}\right) \mid \gamma<\lambda\right\}$.

In short, $c$ is a theory on level $\delta$ of $\mathcal{R H}(T)$ if and only if $c$ satisfies the recovery process on all levels $\gamma \leq \delta$ and $c=c_{\delta}$. It will follow below that $A_{\delta}$ is $\beta$-bounded $\Delta_{0}^{Z F}$ definable (7.1), where $\beta$ is large enough to define the recovery process.

An effective version of the recovery process is woven into the $\Sigma_{1}^{L(\alpha, T)}$ recursive definitions of $\rho_{\delta}$ and $\left\ulcorner A_{\delta}\right\urcorner$ for $0<\delta<\alpha$. The set $\mathcal{L}_{\delta}\left(T_{\delta-}\right)$ is constructible from 
$T_{\delta_{-}}$via the ordinal $\rho_{\delta}$ for all $T_{\delta-} \in A_{\delta-}$, and $\left\ulcorner A_{\delta}\right\urcorner$ is a $\beta$-bounded $\Delta_{0}^{Z F}$ definition of $A_{\delta}$. The definition $\left\ulcorner A_{\delta}\right\urcorner$ specifies the value of $\beta$ and the $\Delta_{0}^{Z F}$ formula.

Stage $0 \quad \mathscr{L}_{0}\left(T_{0-}\right)$ is $\mathscr{L}_{0} ; A_{0}$ is the set of all finitarily consistent, $\omega$-complete theories of $\mathscr{L}_{0}$ extending $T$. Since $\mathscr{L}_{0}$ is recoverable from $T$, the set $A_{0}$ is $\beta$-bounded $\Delta_{0}^{Z F}$ definable with $\beta=0$ and parameter $T$.

Stage $\delta+1 \quad$ Assume the recursion has produced sequences

$$
\left\{\rho_{\gamma} \mid \gamma \leq \delta\right\},\left\{\left\ulcorner A_{\gamma}\right\urcorner \mid \gamma \leq \delta\right\} \in L(\alpha, T)
$$

such that $\left\ulcorner A_{\gamma}\right\urcorner$ is a $\beta$-bounded $\Delta_{0}^{Z F}$ definition of $A_{\gamma}$, and $\mathcal{L}_{\gamma}\left(T_{\gamma-}\right)(\gamma \leq \delta)$ is firstorder definable over

$$
L\left[\rho_{\gamma}, \mathscr{L}_{0} ; T_{\gamma-}\right] .
$$

(The definition of (8.10) follows (7.1).) Consider an arbitrary $T_{\delta} \in A_{\delta}(\delta>0)$. Use the recovery process to construct the unique $T_{\delta-} \in A_{\delta-}$ such that

$$
T_{\delta-} \subseteq T_{\delta} \subseteq \mathscr{L}_{\delta}\left(T_{\delta-}\right) .
$$

The recovery is effective thanks to the sequence $\rho_{\gamma}(\gamma \leq \delta)$. Now $\mathcal{L}_{\delta+1}\left(T_{\delta}\right)$ can be defined as above (8.2) but with an effective twist. Let $S T_{\delta}$ be the set of all $n$-types $(n \geqslant 1)$ of $T_{\delta}$. Since $T$ is weakly scattered, Corollary 3.2 implies

$$
S T_{\delta} \in L\left(\omega_{1}^{T_{\delta}}, T_{\delta}\right)
$$

the least $\Sigma_{1}$ admissible set with $T_{\delta}$ as a member. Let

$$
\gamma_{T_{\delta}}=(\text { least } \gamma)\left[S T_{\delta} \in L\left(\gamma, T_{\delta}\right)\right] .
$$

By Theorem 3.3, the ordinal $\gamma_{T_{\delta}}$, as a function of $T_{\delta}$, is uniformly $\Sigma_{1}$; the same $\Sigma_{1}^{Z F}$ formula singles out $\gamma_{T_{\delta}}$ in $L\left(\omega_{1}^{T_{\delta}}, T_{\delta}\right)$ for every $T_{\delta} \in A_{\delta}$ and for all $\delta$. By Theorem 7.1(i), there is a $\gamma_{\delta}$ such that

$$
\left(\forall T_{\delta} \in A_{\delta}\right)\left[\gamma_{T_{\delta}} \leq \gamma_{\delta}<\alpha\right] .
$$

Hence $S T_{\delta} \in L\left(\gamma_{\delta}, T_{\delta}\right)$ for all $T_{\delta} \in A_{\delta}$. Theorem 7.1(ii) implies that $\gamma_{\delta}$, as a function of $\delta$, has a uniform $\Sigma_{1}$ definition utilizing the parameters occurring in $\left\ulcorner A_{\delta}\right\urcorner$ and the uniform $\Sigma_{1}$ definition of $\gamma_{T_{\delta}}$. Any $n$-type $p(\vec{x}) \in S T_{\delta}$ for any $T_{\delta} \in A_{\delta}$ is constructible from $T_{\delta}$ via some ordinal less than $\gamma_{\delta}$.

A set $\mathcal{P}_{\delta}$ of first-order definitions can be assembled at level $\gamma_{\delta}$ of $L(\alpha, T)$ as follows. Let

$$
\left\{p_{j}^{\mathcal{T}_{\delta}} \mid j \in \mathcal{g}_{\delta}\right\}
$$

be the set of all first-order definitions over $L(\gamma, T)$ for all $\gamma<\gamma_{\delta}$ with parameter $\mathcal{T}_{\delta}$. For each $T_{\delta} \in A_{\delta}$, the object $p_{j}\left(T_{\delta}\right)$ is the set defined by $p_{j}\left(\mathcal{T}_{\delta}\right)$ when the parameter $\mathcal{T}_{\delta}$ is assigned the value $T_{\delta}$. The set (8.15) has a natural well-ordering $W_{\delta}$ definable at level $\gamma_{\delta}$, since each $p_{j}^{\mathcal{J}_{\delta}}$ is specified by its level $\gamma<\gamma_{\delta}$ and its Gödel number $e<\omega$ as a formula of ZF. The type $d_{\delta}\left(\mathcal{T}_{\delta}\right)$, the default type for $\mathcal{T}_{\delta}$, is defined by its action on $T_{\delta} \in A_{\delta}$ :

$$
\begin{aligned}
j\left(T_{\delta}\right) & \left.=\text { (least } j \text { in sense of } W_{\delta}\right)\left[p_{j}\left(T_{\delta}\right) \text { is an } n \text {-type of } T_{\delta}\right] \\
d_{\delta}\left(T_{\delta}\right) & =p_{j\left(T_{\delta}\right)}\left(T_{\delta}\right) .
\end{aligned}
$$


The formula $p_{j}^{\mathcal{T}_{\delta}}$ is a slight variant of $p_{j}\left(\mathcal{T}_{\delta}\right)$ and is defined by its action on $T_{\delta} \in A_{\delta}$.

$$
p_{j}^{T_{\delta}}= \begin{cases}p_{j}\left(T_{\delta}\right) & \text { if } p_{j}\left(T_{\delta}\right) \text { is an } n \text {-type of } T_{\delta} \\ d_{\delta}\left(T_{\delta}\right) & \text { the default type, otherwise }\end{cases}
$$

Let $\mathcal{P}_{\delta}=\left\{p_{j}^{\mathcal{T}_{\delta}} \mid j \in \mathscr{g}_{\delta}\right\}$. Then

1. for all $T_{\delta} \in A_{\delta}$ and $p(\vec{x}) \in S T_{\delta}$, there is a $j \in \mathcal{g}_{\delta}$ such that $p_{j}^{T_{\delta}}$ defines $p(\vec{x})$ at level $\gamma_{\delta}$ of $L(\alpha, T)$, and

2. $p_{j}^{T_{\delta}} \in S T_{\delta}$ for all $T_{\delta} \in A_{\delta}$ and all $j \in \mathcal{g}_{\delta}$.

It can happen for some $T_{\delta} \in A_{\delta}$ and $j, k \in \mathcal{g}_{\delta}$ that $j \neq k$ but $p_{j}^{T_{\delta}}=p_{k}^{T_{\delta}}$. Such repetitions are the price paid to have $\mathcal{P}_{\delta} \in L\left(\gamma_{\delta}+1, T\right)$.

The ordinal $\rho_{\delta+1}<\alpha$ is chosen just large enough to develop the sequence $\rho_{\gamma}(\gamma \leq \delta)$ needed for the recovery of $T_{\delta-}$ from $T_{\delta}(\delta>0)$ and the ordinal $\gamma_{\delta}$ needed to assemble $\mathcal{P}_{\delta}$. The set $\mathscr{L}_{\delta+1}\left(T_{\delta}\right)$ is first-order definable over $L\left[\rho_{\delta+1}, \mathcal{L}_{0} ; T_{\delta}\right]$; its definition begins with $\mathscr{L}_{\delta}\left(T_{\delta-}\right)$, adds the conjunction of all formulas in $p_{j}^{\mathcal{T}_{\delta}}$ for each $p_{j}^{\mathcal{T}_{\delta}} \in \mathcal{P}_{\delta}$, and closes under the finitary operations that generate a fragment of $\mathcal{L}_{\omega_{1}, \omega}$.

To complete stage $\delta+1$, construe $A_{\delta+1}$ to be the set of all $x$ such that the effective version of the recovery process applied to $x$ reports that $x$ is a theory on level $\delta+1$ of $\mathcal{R H}(T)$. The effective version uses the sequence $\rho_{\gamma}(0<\gamma \leq \delta+1)$ to define $\mathscr{L}_{\gamma}\left(T_{\gamma-}\right)$ from $T_{\gamma-}$ for all $T_{\gamma-} \in A_{\gamma-}$. Thus $A_{\delta+1}$ is $\beta$-bounded $\Delta_{0}^{Z F}$ definable with $\beta$ equal to $\rho_{\delta+1}$, and $\left\ulcorner A_{\delta+1}\right\urcorner \in L(\alpha, T)$. The parameter specified by $\left\ulcorner A_{\delta+1}\right\urcorner$ is $T$.

Stage $\lambda$ (limit) Assume for $0<\gamma<\lambda$ that $\mathscr{L}_{\gamma}\left(T_{\gamma-}\right)$ is constructible from $T_{\gamma-}$ via $\rho_{\gamma}$ for all $T_{\gamma_{-}} \in A_{\gamma_{-}}$. Use the effective version of the recovery process to define $A_{\lambda}$ as a $\beta$-bounded $\Delta_{0}^{Z F}$ class. For $T_{\gamma} \in A_{\lambda}$, effectively recover the unique sequence $T_{\gamma}(\gamma<\lambda)$ such that $T_{\lambda}$ is $\cup\left\{T_{\gamma} \mid \gamma<\lambda\right\}$, and then define $\mathcal{L}_{\lambda}\left(T_{\lambda}\right)$ to be $\cup\left\{\mathcal{L}_{\gamma}\left(T_{\gamma_{-}}\right) \mid 0<\gamma<\lambda\right\}$.

Makkai [10] showed that if $T$ is a counterexample to Vaught's Conjecture, then $T$ has a model of cardinality $\omega_{1}$ that is $\mathcal{L}_{\infty, \omega}$ equivalent to a countable model. The following are variants of his results.

Suppose $A$ is a countable $\Sigma_{1}$ admissible set and $T \in A$. Assume $T \subseteq \mathscr{L}_{0}, \mathscr{L}_{0}$ is a countable fragment of $\mathscr{L}_{\omega_{1}, \omega}$, and $\mathscr{L}$ is a countable first-order language. Also assume every symbol of $\mathcal{L}$ is mentioned in $T$ so that $\mathcal{L}$ is recoverable from $T$. Let $\mathscr{L}^{\prime}$ denote an arbitrary fragment of $\mathscr{L}_{\omega_{1}, \omega}$ that extends $\mathscr{L}$, and $T^{\prime}$ an arbitrary finitarily consistent, $\omega$-complete theory contained in $\mathcal{L}^{\prime}$ and extending $T$. Call $T$ weakly scattered in $A$ if and only if $S T^{\prime} \in A$ for all $T^{\prime} \in A$. According to Theorem 3.3, we have the following.

Theorem 8.1 Suppose $\mathcal{A}$ is a countable model of $T$. Assume $T$ is weakly scattered in $L\left(\omega_{1}^{T, \mathcal{A}},\langle T, \mathcal{A}\rangle\right)$, and

$$
\operatorname{sr}(\mathcal{A}) \geq \omega_{1}^{T, \mathcal{A}} .
$$

Then $\mathcal{A}$ is $\mathcal{L}_{\infty, \omega}$ equivalent to a model of $T$ of cardinality $\omega_{1}$.

Proof Let $\alpha=\omega_{1}^{T, \mathcal{A}}$. Thus $\omega_{1}^{\mathcal{A}}=\alpha$, since $\omega_{1}^{\mathcal{A}}+1 \geq \operatorname{sr}(\mathcal{A})$. Let $T_{\beta}^{\mathcal{A}}(\beta \leq \operatorname{sr}(\mathcal{A}))$ be the $\mathrm{Scott}$ analysis of $\mathcal{A}$ as defined in Section 2. By Theorem 3.3, $S T_{\beta}^{\mathcal{A}} \in L(\alpha,\langle T, \mathcal{A}\rangle)$ 
(and so $T_{\beta}^{\mathcal{A}}$ has a countable atomic model) for all $\beta$ such that $\beta+1<\operatorname{sr}(\mathcal{A})$. The set $Z$ is $\Sigma_{1}^{L(\alpha,\langle T, \mathcal{A}\rangle)}$ and consists of the following sentences:

(Z1) the atomic diagram (in the sense of $\left.\mathscr{L}_{\omega_{1}, \omega}\right)$ of $L(\alpha,\langle T, \mathcal{A}\rangle)$;

(Z2) $\underline{d}$ is a countable ordinal and $\underline{d} \geq \delta\left(\right.$ all $\left.\delta<\omega_{1}^{T, \mathcal{A}}\right)$;

(Z3) $\forall y\left[y<\underline{d} \rightarrow T_{y}^{\mathcal{A}}\right.$ has a countable atomic model $]$;

(Z4) axioms of $\Sigma_{1}$ admissibility.

The set $Z$ is consistent since it can be modeled by $V$ (the real world). Every model of $Z$ is an end extension of $L(\alpha,\langle T, \mathcal{A}\rangle)$. Let $M$ be a model of $Z$ that omits $\alpha$. Thus $M$ has nonstandard ordinals greater than every ordinal less than $\alpha$. Hence $\operatorname{sr}(\mathcal{A}) \geq \alpha$ in $V$ and $\alpha \notin M$, so $\operatorname{sr}(\mathcal{A}) \geq \gamma$ for some nonstandard $\gamma \in M$.

Now work inside $M$. Let $T_{\delta}^{\mathcal{A}}(\delta \leq \gamma)$ be the Scott analysis of $\mathcal{A}$ up to level $\gamma$. Choose a nonstandard $\beta<\gamma$. Then $T_{\beta}^{\mathcal{A}}$ has a countable atomic model $\mathcal{A}_{\beta}$. There is a map

$$
i_{\beta \gamma}: \mathcal{A}_{\beta} \rightarrow \mathcal{A}
$$

that is elementary with respect to all formulas of $\mathcal{L}_{\beta}^{\mathcal{A}}$ (defined in Section 2). Note that $i_{b y}$ is not onto, since $\mathcal{A}_{\beta}$ is not isomorphic to $\mathcal{A}$ in $M$.

But $\mathcal{A}_{\beta}$ is isomorphic to $\mathcal{A}$ in $V$. Now $\omega_{1}^{\mathcal{A}_{\beta}} \leq \alpha$ since $\alpha \notin M$; also $\operatorname{sr}\left(\mathcal{A}_{\beta}\right) \geq \delta$ for all $\delta<\alpha$. Hence $\operatorname{sr}\left(\mathcal{A}_{\beta}\right) \geq \alpha$, and so $\omega_{1}^{\mathcal{A}_{\beta}} \geq \alpha$. Thus both $\mathcal{A}_{\beta}$ and $\mathcal{A}_{\text {are }}$ homogeneous models of $T_{\alpha}^{\mathcal{A}}$ by (2.6). To see they realize the same types of $T_{\alpha}^{\mathcal{A}}$, choose $p_{\alpha} \in S T_{\alpha}^{\mathcal{A}}$ and first suppose $\mathcal{A}_{\beta} \models p_{\alpha}(\bar{b})$. In $M$, note that $\mathcal{A}_{\beta} \models p_{\beta}(\bar{b})$ for some type $p_{\beta}$ of $T_{\beta}^{\mathcal{A}}$ and that $\mathcal{A} \models p_{\gamma}\left(i_{\beta \gamma}(\bar{b})\right)$ for some type $p_{\gamma}$ of $T_{\gamma}{ }^{\mathcal{A}}$. Then

$$
p_{\alpha} \subseteq p_{\beta} \subseteq p_{\gamma},
$$

since $i_{\beta \gamma}$ is $\mathcal{L}_{\beta}^{\mathcal{A}}$ elementary. Hence $\mathcal{A} \models p_{\alpha}\left(i_{\beta \gamma}(\bar{b})\right)$. It follows that

$$
i_{\beta \gamma} \text { is } \mathcal{L}_{\omega_{1}, \omega} \text { elementary, }
$$

since the types of $T_{\alpha}^{\mathcal{A}}$ realized in $\mathcal{A}_{\beta}$ are atoms of $\mathcal{L}_{\omega_{1}, \omega}$.

Now suppose $\mathcal{A} \models p_{\alpha}(\bar{a})$. In $M$, the tuple $\bar{a}$ realizes $p_{\gamma}$ in $\mathcal{A}$, a type of $T_{\gamma}^{\mathcal{A}}$. Choose a nonstandard $\delta<\beta$. Let $p_{\beta}$ be the restriction of $p_{\gamma}$ to $\mathcal{L}_{\beta}^{A}$, and let $p_{\delta}$ be the restriction to $\mathcal{L}_{\delta}^{\mathcal{A}}$. Then $p_{\alpha} \subseteq p_{\delta} \subseteq p_{\beta} \subseteq p_{\gamma}$. So

$$
\mathcal{A} \vDash \exists \bar{x} p_{\delta}(\bar{x}) \text {. }
$$

But then $\exists \bar{x} p_{\delta}(\bar{x}) \in T_{\delta+1} \subseteq T_{\beta}$, so $p_{\delta}$, hence $p_{\alpha}$, is realized in $\mathcal{A}_{\beta}$.

Thanks to the above there exist structures $\mathscr{B}_{0}$ and $\mathscr{B}_{1}$, both isomorphic to $\mathcal{A}$, such that $\mathcal{B}_{0} \subsetneq \mathcal{B}_{1}$ and the inclusion map $i$ is $\mathscr{L}_{\omega_{1}, \omega}$ elementary. A strictly expanding $\mathcal{L}_{\omega_{1}, \omega}$ elementary chain $\mathscr{B}_{\delta}\left(\delta \leq \omega_{1}\right)$ is defined by iterating $i$. For $\delta<\omega_{1}$, assume $\mathscr{B}_{\delta}$ is isomorphic to $\mathcal{A}$. Then enlarge $\mathscr{B}_{\delta}$ to $\mathcal{B}_{\delta+1}$, another copy of $\mathcal{A}$. For limit $\lambda \leq \omega_{1}$, let $\mathscr{B}_{\lambda}$ be the union of the $\mathscr{B}_{\delta} \mathrm{s}(\delta<\lambda)$. $\mathscr{B}_{\omega_{1}}$ is an $\mathscr{L}_{\omega_{1}, \omega}$ elementary extension of $\mathscr{B}_{0}$, hence $\mathcal{L}_{\omega_{1}, \omega}$-equivalent to $\mathcal{A}$, consequently $\mathcal{L}_{\infty, \omega}$-equivalent to A.

Corollary 8.2 Suppose $T$ is weakly scattered. For each $\beta<\omega_{1}^{T}$, assume $T$ has a model of Scott rank $\geq \beta$. Then $T$ has a countable model A such that

$$
\operatorname{sr}(\mathcal{A}) \geq \omega_{1}^{T, \mathcal{A}}=\omega_{1}^{T},
$$


and every such $\mathcal{A}$ is $\mathscr{L}_{\infty, \omega}$ equivalent to a model of $T$ of cardinality $\omega_{1}$.

\section{Bounds on Weakly Scattered Theories}

Once again let $\mathscr{L}_{0}$ be a countable fragment of $\mathcal{L}_{\omega_{1}, \omega}$ for some countable first-order language $\mathscr{L}$ and $T \subseteq \mathscr{L}_{0}$ a weakly scattered theory with a model. Assume $L(\alpha, T)$ is $\Sigma_{1}$ admissible. Consider $B_{\alpha}$, a $\Delta_{1}^{L(\alpha, T)}$ set of sentences designed so that every model of $B_{\alpha}$ constitutes a node on level $\alpha$ of $\mathcal{R H}(T)$, the raw hierarchy for $T$. The axioms of $B_{\alpha}$ are

1. $T \subseteq T_{0}$ and $T_{0}$ is a finitarily consistent, $\omega$-complete theory of $\mathscr{L}_{0}$,

2. $T_{\delta}$ has a nonprincipal $n$-type for some $n$ (all $\delta<\alpha$ ),

3. $T_{\delta} \subseteq T_{\delta+1}$ and $T_{\delta+1}$ is a finitarily consistent, $\omega$-complete theory of $\mathcal{L}_{\delta+1}\left(T_{\delta}\right)$ (all $\delta<\alpha$ ),

4. $T_{\lambda}=\cup\left\{T_{\delta} \mid \delta<\lambda\right\}$ and $\mathcal{L}_{\lambda}\left(T_{\lambda}\right)=\cup\left\{\mathcal{L}_{\delta}\left(T_{\delta-}\right) \mid \delta<\lambda\right\}$ (all limit $\lambda<\alpha$ ).

Then $B_{\alpha}$ is $\Delta_{1}^{L(\alpha, T)}$ because Section 8 shows how to construct $\mathcal{L}_{\delta}\left(T_{\delta-}\right)$ from $T_{\delta-}$ via the ordinal $\rho_{\delta}$ defined by a $\Sigma_{1}^{L(\alpha, T)}$ recursion on $\delta<\alpha$.

Sets $\mathcal{P}_{\delta}$ and $\mathscr{g}_{\delta}$ were defined just after (8.14). Define ' $p$ is on level $\delta$ ' by

$$
p=p_{j}^{\mathcal{T}_{\delta}} \text { for some } j \in \mathscr{g}_{\delta} .
$$

A split at level $\delta$ is a sentence of the form $p$ is on level $\delta$, and there exist $r$ and $r^{\prime}$ on level $\delta+1$ such that $r \neq r^{\prime}$ and both $r$ and $r^{\prime}$ extend $p$. The sentence in abbreviated form is $\left\langle p, r, r^{\prime}\right\rangle$. A split is a sentence of $\mathcal{L}_{\omega_{1}, \omega} \cap L(\alpha, T)$, because $\mathcal{P}_{\delta}$, $\mathcal{P}_{\delta+1} \in L(\alpha, T)$. The triple $\left\langle p, r, r^{\prime}\right\rangle$ is a $k$-split if $p$ has arity $k$. Let $K$ be a set of $k$-splits. The set $K$ is unbounded if and only if

$$
\forall \beta<\alpha(\exists \delta>\beta)[K \text { has a } k \text {-split on level } \delta] .
$$

$K$ has the predecessor property if and only if there is a partial function $f(p, \gamma)$ such that if $\gamma<\delta$ and $\left\langle p, r, r^{\prime}\right\rangle \in K$ and asserts $p$ splits at level $\delta$, then $f(p, \gamma)$ is defined and belongs to $g_{\gamma}$, and

$$
B_{\alpha} \vdash\left[\left\langle p, r, r^{\prime}\right\rangle \longrightarrow\left(p_{f(p, \gamma)}^{\mathcal{T}_{\gamma}} \text { is extended by } p\right)\right] .
$$

If such an $f$ exists, then there is one that is $\Sigma_{1}^{L(\alpha, T)}$ definable, since the $\Delta_{1}^{L(\alpha, T)}$ definability of $B_{\alpha}$ implies the deduction claimed by (9.3) can be found in $L(\alpha, T)$.

The effective $k$-splitting hypothesis holds for $T$ at $\alpha$ if and only if there exists an unbounded $\Delta_{1}^{L(\alpha, T)}$ set $K$ of $k$-splits such that $K$ has the predecessor property and $B_{\alpha} \cup K$ is consistent (in the sense of $\mathcal{L}_{\omega_{1}, \omega}$ restricted to $L(\alpha, T)$ )) if $B_{\alpha}$ is. Consider Makkai's example [11] (also [8]) mentioned in Section 1. It can be formulated as a fragment $\mathscr{L}_{0}$ and a theory $T_{M} \subseteq \mathscr{L}_{0}$, both arithmetically definable, with the following properties:

(1) $T_{M}$ is not weakly scattered;

(2) every countable model $\mathcal{A}$ of $T_{M}$ has Scott rank at most $\omega_{1}^{\mathcal{A}}$;

(3) for every countable $\Sigma_{1}$ admissible $L(\alpha)$, there is a countable model $\mathcal{A}$ of $T_{M}$ such that $\omega_{1}^{\mathcal{A}}=\alpha=\operatorname{sr}(\mathcal{A})$.

Despite (1) it is possible to develop a crude hierarchy for $T_{M}$ with a superficial resemblance to the raw hierarchy $\mathcal{R} \mathcal{H}(T)$ of Section 8 . For $\delta<\omega_{1}$ put theory $T^{\prime} \supseteq T_{M}$ on level $\delta$ if there exists a countable model $\mathcal{A}$ of $T_{M}$ such that $\operatorname{sr}(\mathcal{A})=\delta$ and $T^{\prime}=T_{\operatorname{sr}(\mathcal{A})}^{\mathcal{A}}$ (as defined in Section 2). Since $T_{M}$ is not weakly scattered, it is not possible to give 
a bounded description of all types associated with all theories on level $\delta$, as was done with $\mathcal{P}_{\delta}$ in Section 8. Nonetheless, some of the types on level $\delta$ have properties that lend credence to the effective $k$-splitting hypothesis. The model $\mathcal{A}$ of (3) above is a tree with $\omega$ many levels and infinite paths. Some nodes of $\mathcal{A}$ have foundation rank (fr) $<\infty$. Foundation rank $\omega \delta+m$ corresponds to atoms of $T_{\omega_{1}^{\mathcal{A}}}^{\mathcal{A}}$ of rank $\delta$. Associated with level $\delta$ of $\mathcal{C} \mathscr{H}\left(T_{M}\right)$, the crude hierarchy for $T_{M}$, are types of the form

$$
x \text { is on level } \delta \text { of } \mathcal{A} \text { and } \operatorname{fr}(x) \geq \omega \delta+m
$$

that split on level $\delta+1$ of $\mathcal{C H}(T)$. On level $\gamma<\delta$, (9.4) has a predecessor similar to (9.4) with $\delta$ replaced by $\gamma$.

Theorem 9.1 Suppose $T$ is weakly scattered and $L(\alpha, T)$ is countable and $\Sigma_{2}$ admissible. For each $\beta<\alpha$, suppose $T$ has a model of Scott rank at least $\beta$. If for some $k$, the effective $k$-splitting hypothesis holds for $T$ at $\alpha$, then $T$ has a countable model $\mathcal{A}$ such that

$$
\omega_{1}^{\mathcal{A}}=\alpha \text { and } \operatorname{sr}(\mathcal{A})=\alpha+1 \text {. }
$$

Proof By Barwise Compactness, $T$ has a model $\mathcal{A}$ such that $L(\alpha,\langle T, \mathcal{A}\rangle)$ is $\Sigma_{1}$ admissible and $\operatorname{sr}(\mathcal{A}) \geq \alpha$. Then $\operatorname{rtr}(\mathcal{A}) \geq \alpha$ by (8.5) and so $B_{\alpha}$ is consistent. Let $K$ be an unbounded $\Delta_{1}^{L(\alpha, T)}$ set of $k$-splits with a $\Sigma_{1}^{L(\alpha, T)}$ predecessor function $f(\gamma, p)$. A model of $B_{\alpha} \cup K$ is constructed so that $T_{\alpha}$ has a nonprincipal type $q_{\alpha}$ and the structure

$$
L\left[\alpha, T ; T_{\alpha}, q_{\alpha}\right]
$$

is $\Sigma_{1}$ admissible with respect to $\Sigma_{1}$ formulas that include $T_{\alpha}$ and $q_{\alpha}$ as atomic predicates. Then, as in the type omitting proof of Theorem 6.1, $T$ has a model $\mathcal{A}_{1}$ realizing $q_{\alpha}$ and such that $\omega_{1}^{\mathcal{A}_{1}}=\alpha$. The universe of (9.5) is the result of iterating first-order definability through the ordinals less than $\alpha$ starting with $T$ and with $T_{\alpha}, q_{\alpha}$ as additional atomic predicates. The construction of (9.5) is Henkinesque and gradually decides all sentences of rank less than $\alpha$ in a standard language $\mathcal{L}_{\alpha, T} \in \Delta_{1}^{L(\alpha, T)}$ that names all elements of (9.5) and is able to express how each one is defined from those of lower definability rank. The language $\mathcal{L}_{\alpha, T}$ does not have symbols $T_{\alpha}$ or $q_{\alpha}$ but does have symbols $T_{\beta}$ and $q_{\beta}$ for all $\beta<\alpha$. There is one twist. The $\Sigma_{1}$ admissibility of (9.5) is not obtained by an effective type omitting argument that omits $\alpha$ as in the proof of Theorem 6.1 but by direct manipulation of ranked sentences of $\mathcal{L}_{\alpha, T}$. The twist avoids Henkin constants.

Let $S_{n}$ be the set of sentences chosen by the end of stage $n . S_{n}$ will be $\Sigma_{2}^{L(\alpha, T)}$ definable. $S_{0}$ requires some preparation. Consider $p_{j}^{\mathcal{T}_{\gamma}}$ for some $j \in g_{\gamma} \cdot p_{j}^{\mathcal{T}_{\gamma}}$ is said to be $K$-unbounded if the set of all $\delta$ such that

$$
\exists\left\langle p, r, r^{\prime}\right\rangle\left[\left\langle p, r, r^{\prime}\right\rangle \in K, p \text { is on level } \delta, f(p, \gamma)=p_{j}^{\mathcal{T}_{\gamma}}\right]
$$

is unbounded in $\alpha$. Thus $B_{\alpha} \cup K$ implies $p_{j}^{\mathcal{T}_{\gamma}}$ has unboundedly many extensions that split in $K . K$-unboundedness is a $\Pi_{2}^{L(\alpha, T)}$ property. $K$-bounded means "not $K$-unbounded'.

Claim 9.2 For all $\gamma$ there is a $K$-unbounded type on level $\gamma$.

Proof of claim Suppose not. Then for each $j \in g_{\gamma}$, there is a least $\beta_{j}$ such that for all $\delta \geq \beta_{j},(9.6)$ is false. The ordinal $\beta_{j}$, as a function of $j$, is $\Sigma_{2}^{L(\alpha, T)}$, hence 
bounded by some $\beta_{\infty}<\alpha$. But then $K$ is bounded by $\beta_{\infty}$. A set $U \subseteq K$ is said to be bounded if

$$
\exists \beta<\alpha(\forall \delta>\beta)[U \text { does not have a } k \text {-split on level } \delta] .
$$

Definition 9.3 (Definition of $S_{0}$ ) Start with $B_{\alpha} \cup K$. Add

1. sentences of $\mathcal{L}_{\alpha, T}$ that express how each element of (9.5) is defined from elements of lower rank;

2. $q_{\beta}$ is a type on level $\beta(\beta<\alpha)$;

3. $q_{\beta}$ is extended by $q_{\gamma}(\beta<\gamma<\alpha)$;

4. $q_{\beta} \neq p(\beta<\alpha$ and $p$ is $K$-bounded $)$.

Note that ' $q_{\beta}$ is a type on level $\beta$ ' is a ranked sentence, in particular, a disjunction, by the remarks following (8.14).

$S_{0}$ is $\Sigma_{2}^{L(\alpha, T)}$ definable since $K$-boundedness is $\Sigma_{2}^{L(\alpha, T)}$. To check the consistency of $S_{0}$, let $M$ be a model of $B_{\alpha} \cup K$ that specifies the structure of $L\left(\alpha, T ; T_{\alpha}\right)$ but says nothing about $q_{\gamma}$ for any $\gamma<\alpha$. Fix $\tau<\alpha$. Suppose $\gamma<\tau$; then $M$ can be interpreted as a model of those sentences in $S_{0}$ that mention $q_{\gamma}$ only for $\gamma<\tau$. Choose a $K$-unbounded $p_{\tau}$ on level $\tau$ with the aid of Claim 9.2. Define

$$
\begin{aligned}
U_{\tau} & =\left\{s \mid \exists t, t^{\prime}\left[\left\langle s, t, t^{\prime}\right\rangle \in K\right] \text { and } f(s, \tau)=p_{\tau}\right\}, \\
U_{\gamma}^{r} & =\left\{s \mid s \in U_{\tau} \wedge f(s, \gamma)=r\right\}(\gamma<\tau) .
\end{aligned}
$$

Fix $\gamma<\tau$. There must be a $K$-unbounded $r$ on level $\gamma$. Suppose not. Then $U_{\gamma}^{r}$ is bounded for every $r$ on level $\gamma$. But

$$
U_{\tau}=\cup\left\{U_{\gamma}^{r} \mid r \text { is on level } \gamma\right\} .
$$

Hence $U_{\tau}$ is bounded by the $\Sigma_{2}$ admissibility argument used to prove (9.2), and so $p_{\tau}$ is $K$-bounded.

For each $\gamma<\tau$, choose a $K$-unbounded $r_{\gamma}$ on level $\gamma$. To see that for each $\gamma<\tau$,

$$
B_{\alpha} \cup K \vdash r_{\gamma} \text { is extended by } p_{\tau},
$$

let $s \in U_{\gamma}^{r_{\gamma}}$. Then $s \in U_{\tau}$. Assume $B_{\alpha} \cup K$. Then $s$ extends $f(s, \tau)=p_{\tau}$ and $s$ extends $f(s, \gamma)=r_{\gamma}$. Hence $p_{\tau}$ extends $r_{\gamma}$.

It follows from (9.10) that

$$
B_{\alpha} \cup K \vdash r_{\gamma_{1}} \text { is extended by } r_{\gamma_{2}}
$$

when $\gamma_{1}<\gamma_{2}<\tau$. Now $M$, as promised above, can be interpreted as a model of that part of $S_{0}$ that mentions $q_{\gamma}$ only for $\gamma<\tau$ by setting the interpretation of $q_{\gamma}$ in $M$ equal to that of $r_{\gamma}$.

Definition 9.4 (Definition of $S_{n+1}$ ) Assume $S_{n}$ is consistent and $\Sigma_{2}^{L(\alpha, T)}$. There are two cases.

Case (a) Suppose $\mathcal{F}=\vee\left\{\mathcal{F}_{i} \mid i \in I\right\}$ is a ranked sentence such that $S_{n} \cup\{\mathcal{F}\}$ is consistent. $S_{n+1}$ is $S_{n} \cup\left\{\mathcal{F}_{i^{\prime}}\right\}$ for some $i^{\prime} \in I$ such that $S_{n} \cup\left\{\mathcal{F}_{i^{\prime}}\right\}$ is consistent. 
Case (b) The purpose of this case is to establish $\Delta_{0}$ bounding, hence $\Sigma_{1}$ replacement, for (9.5). Let $\mathscr{D}(x, y)$ be a $\Delta_{0}^{Z F}$ formula with constants naming elements of (9.5). Fix $\rho<\alpha$, and regard $\mathscr{D}(x, y)$ as possibly defining a many-valued function $d(x)$ from $\rho$ into $\alpha$ that is $\Delta_{0}$ in the sense of (9.5). For each $\delta<\rho$, define

$$
H_{\delta}=\{\neg D(\delta, \gamma) \mid \gamma<\alpha\} .
$$

Subcase (b1) Suppose there is a $\delta<\rho$ such that $S_{n} \cup H_{\delta}$ is consistent. Let $\delta^{\prime}$ be such a $\delta$, and put $S_{n+1}$ equal to $S_{n} \cup H_{\delta^{\prime}}$. Then $d\left(\delta^{\prime}\right)$ will be undefined.

Subcase (b2) Suppose (b1) fails. Then for each $\delta<\rho$,

$$
S_{n} \vdash \vee\{D(\delta, \gamma) \mid \gamma<\alpha\}
$$

so by Barwise Compactness there is a $c(\delta)<\alpha$ such that

$$
S_{n} \vdash \vee\{D(\delta, \gamma) \mid \gamma<c(\delta)\} .
$$

$c(\delta)$ can be defined via deductions from $S_{n}$ as a $\Sigma_{2}^{L(\alpha, T)}$ function of $\delta$. Let $c$ be $\sup \{c(\delta) \mid \delta<\rho\}$. Then $c<\alpha$ and $d(\delta)(\delta<\rho)$ will be bounded by $c$.

Define $S=\cup\left\{S_{n} \mid n<\omega\right\}$. By Case (a), $S$ specifies (9.5). $q_{\alpha}$ is a nonprincipal type of $T_{\alpha}$, because for every $\beta<\alpha, S_{0}$ and Claim 9.2 compel $q_{\beta}$ to be $K$-unbounded and consequently to split. (An instance of Case (a) results in the choice of a $K$ unbounded $p$ such that $\left(q_{\beta}=p\right.$ ) belongs to $S$.) By Case (b), (9.5) is $\Sigma_{1}$ admissible. It follows, as in the proof of Theorem 6.1, that $T$ has a model $\mathcal{A}_{1}$ that realizes $q_{\alpha}$ and such that $\omega_{1}^{\mathcal{A}_{1}}=\alpha$. Hence $\operatorname{sr}(\mathcal{A})=\alpha+1$.

Corollary 9.5 (Bounding) $\quad$ Suppose $T$ is weakly scattered and for some $k$ satisfies the effective $k$-splitting hypothesis at $\alpha$. If $L(\alpha, T)$ is $\Sigma_{2}$ admissible and

$$
(\forall \text { countable } \mathcal{A})\left[\mathcal{A} \models T \longrightarrow \operatorname{sr}(\mathcal{A}) \leq \omega_{1}^{\mathcal{A}}\right],
$$

then

$$
(\exists \beta<\alpha)(\forall \mathcal{A})[\mathcal{A} \models T \longrightarrow \operatorname{sr}(\mathcal{A})<\beta]
$$

\section{Further Results and Open Questions}

Weakening the assumption of effective $k$-splitting in Section 9 is under study. At this writing it appears likely that the predecessor (9.3) property can be dropped from the assumption: all that is needed is an unbounded $\Delta_{1}^{L(\alpha, T)}$ set of $k$-splits consistent with $B_{\alpha}$; then the existence of a predecessor function can be proved. (See Goddard [4].) There is a price to pay: the type structure $p_{j}^{\tau_{\delta}}(\delta<\alpha)$ of a weakly scattered theory $T$ has to be treated with greater delicacy. A further weakening, less likely but more than plausible, is to rule out the existence of RN-models of $T$. Call $\mathcal{A}$ an $R N$-model of $T$ if and only if (i) $\operatorname{sr}(\mathcal{A})=\omega_{1}^{\mathcal{A}}$, (ii) $T_{\omega_{1}^{\mathcal{A}}}^{\mathcal{A}}$ is $\omega$-categorical, and (iii) for each $n$ there is a $\beta<\omega_{1}^{\mathcal{A}}$ such that each principal $n$-type of $T_{\omega_{1}^{\mathcal{A}}}^{\mathcal{A}}$ of arity $n$ is generated by a formula of rank less than $\beta$. ( $T_{\omega_{1}^{\text {A }}}^{\mathcal{A}}$ is defined in Section 2.) Makkai [11] produces an $\mathcal{A}$ that satisfies (i) and (ii) but not (iii).

It appears that iterated forcing has a role to play above and also in the construction of an $\alpha$-saturated model of $T$ when $T$ is weakly scattered and has countable models of unbounded Scott rank. But that is another story. (See Chan [3].) 


\section{Notes}

1. [15] was a hasty writeup of a talk given at the 1971 meeting of the International Congress of Logic, Methodology and Philosophy of Science. Some details absent from [15] but needed here are presented below.

2. Strictly speaking, the relativization is to the transitive closure of $\mathcal{A}$.

3. As final corrections were being made to the galleys, it was discovered that Theorem 4.9 here is also reproved as Theorem 3.8, p. 84 of Baldwin [1] in this special issue.

\section{References}

[1] Baldwin, J., "The Vaught Conjecture: Do Uncountable Models Count?," Notre Dame Journal of Formal Logic, vol. 48 (2007), pp. 79-92 (electronic). 30

[2] Barwise, J., Admissible Sets and Structures. An Approach to Definability Theory, Perspectives in Mathematical Logic. Springer-Verlag, Berlin, 1975. Zbl 0316.02047. MR 0424560. 9

[3] Chan, A., Models of High Rank for Weakly Scattered Theories, Ph.D. thesis, Massachusetts Institute of Technology, 2006. 29

[4] Goddard, C., Improving a Bounding Result for Weakly-Scattered Theories, Ph.D. thesis, Massachusetts Institute of Technology, 2006. 29

[5] Grilliot, T. J., "Omitting types: Application to recursion theory," The Journal of Symbolic Logic, vol. 37 (1972), pp. 81-89. Zbl 0244.02016. MR 0344099. 6, 14

[6] Harnik, V., and M. Makkai, "A tree argument in infinitary model theory," Proceedings of the American Mathematical Society, vol. 67 (1977), pp. 309-14. Zbl 0384.03019. MR 0472506. 7, 14

[7] Keisler, H. J., Model Theory for Infinitary Logic. Logic with Countable Conjunctions and Finite Quantifiers, vol. 62 of Studies in Logic and the Foundations of Mathematics, North-Holland Publishing Co., Amsterdam, 1971. Zbl 0222.02064. MR 0344115. 6, 14

[8] Knight, J. F., and J. M. Young, “Computable structures of rank $\omega_{1}^{C K}$," preprint, 2004. 6,26

[9] Knight, R. W., “The Vaught Conjecture: A Counterexample,” manuscript, 2002. http://www.maths.ox.ac.uk/ knight/stuff/example2.ps 7

[10] Makkai, M., "An 'admissible' generalization of a theorem on countable $\Sigma_{1}^{1}$ sets of reals with applications," Annals of Pure and Applied Logic, vol. 11 (1977), pp. 1-30. Zbl 0376.02031. MR 0491142. 9, 24

[11] Makkai, M., "An example concerning Scott heights," The Journal of Symbolic Logic, vol. 46 (1981), pp. 301-18. Zbl 0501.03018. MR 613284. 6, 7, 19, 26, 29

[12] Millar, J., and G. E. Sacks, "Models with high Scott rank," in preparation. 7 
[13] Morley, M., “The number of countable models," The Journal of Symbolic Logic, vol. 35 (1970), pp. 14-18. Zbl 0196.01002. MR 0288015. 7, 11, 15

[14] Nadel, M., "Scott sentences and admissible sets," Annals of Pure and Applied Logic, vol. 7 (1974), pp. 267-94. Zbl 0301.02050. MR 0384471. 7, 8, 9

[15] Sacks, G. E., "On the number of countable models," pp. 185-95 in Southeast Asian Conference on Logic (Singapore, 1981), vol. 111 of Studies in Logic and the Foundations of Mathematics, North-Holland, Amsterdam, 1983. Zbl 0563.03015. MR 723338. 5, 7, $8,9,11,18,30$

[16] Sacks, G. E., Higher Recursion Theory, Perspectives in Mathematical Logic. SpringerVerlag, Berlin, 1990. Zbl 0716.03043. MR 1080970. 6

[17] Scott, D., "Logic with denumerably long formulas and finite strings of quantifiers," pp. 329-41 in Theory of Models (Proceedings of the 1963 International Symposium, Berkeley), North-Holland, Amsterdam, 1965. Zbl 0166.26003. MR 0200133. 6, 8

[18] Steel, J. R., “On Vaught's conjecture,” pp. 193-208 in Cabal Seminar 76-77 (Proceedings of the Caltech-UCLA Logic Seminar, 1976-77), vol. 689 of Lecture Notes in Mathematics, Springer, Berlin, 1978. Zbl 0403.03027. MR 526920. 7, 19

\section{Acknowledgments}

Many thanks to Julia Knight for her patience and encouragement.

Deprtment of Mathematics

Harvard University

Cambridge MA 02138

and

Department of Mathematics

Massachusetts Institute of Technology

77 Massachusetts Ave

Cambridge MA 02139-4307

sacks@math.harvard.edu

sacks@math.mit.edu 